\title{
Chronic skin inflammation accelerates macrophage cholesterol crystal formation and atherosclerosis
}

\author{
Yvonne Baumer, ${ }^{1}$ Qimin Ng, ${ }^{1}$ Gregory E. Sanda, ${ }^{1}$ Amit K. Dey, ${ }^{1}$ Heather L. Teague, ${ }^{1}$ \\ Alexander V. Sorokin, ${ }^{1}$ Pradeep K. Dagur, ${ }^{2}$ Joanna I. Silverman, ${ }^{1}$ Charlotte L. Harrington, ${ }^{1}$ \\ Justin A. Rodante, ${ }^{1}$ Shawn M. Rose, ${ }^{1}$ Nevin J. Varghese, ${ }^{1}$ Agastya D. Belur, ${ }^{1}$ Aditya Goyal, \\ Joel M. Gelfand, ${ }^{3,4}$ Danielle A. Springer, ${ }^{5}$ Christopher K.E. Bleck, ${ }^{6}$ Crystal L. Thomas, ${ }^{7}$ \\ Zu-Xi Yu, ${ }^{8}$ Mårten C.G. Winge, ${ }^{9}$ Howard S. Kruth, ${ }^{10}$ M. Peter Marinkovich,,${ }^{9,11}$ Aditya A. Joshi, ${ }^{1}$ \\ Martin P. Playford, ${ }^{1}$ and Nehal N. Mehta ${ }^{1}$ \\ 15ection of Inflammation and Cardiometabolic Diseases and ${ }^{2} F l o w$ Cytometry Core, National Heart, Lung, and Blood \\ Institute (NHLBI), NIH, Bethesda, Maryland, USA. ${ }^{3}$ Department of Dermatology, Perelman School of Medicine, ${ }^{4}$ The \\ Center for Clinical Epidemiology and Biostatistics, University of Pennsylvania, Philadelphia, Pennsylvania, USA. ${ }^{5}$ Murine \\ Phenotyping Core, ${ }^{6}$ Electron Microscopy Core Facility, ${ }^{7}$ Comparative Medicine Branch, National Institute of Allergy and \\ Infectious Diseases (NIAID), and ${ }^{8}$ Pathology Core Facility, Department of Health and Human Services, NHLBI, NIH, \\ Bethesda, Maryland, USA. ${ }^{9}$ Program in Epithelial Biology, Stanford University School of Medicine, Stanford, California, \\ USA. ${ }^{10}$ Section of Experimental Atherosclerosis, NHLBI, NIH, Bethesda, Maryland, USA. "11Dermatology Service, Veterans \\ Affairs Medical Center, Palo Alto, California, USA.
}

Authorship note: Y. Baumer and Q. Ng contributed equally to this work.

Conflict of interest: N.N. Mehta is a full-time US Government employee and receives research grants from Abbvie, Janssen, Novartis Corp, and Celgene. J.M. Gelfand served as a consultant for Coherus (DSMB), Dermira, Janssen Biologics, Merck (DSMB), Novartis Corp, Regeneron, Sanofi, and Pfizer Inc., receiving honoraria; receives research grants (to the Trustees of the University of Pennsylvania) from Abbvie, Janssen, Novartis Corp, Regeneron, Sanofi, Celgene, and Pfizer Inc.; and received payment for continuing medical education work related to psoriasis that was supported indirectly by Lilly and Abbvie. M.C.C. Winge and M.P. Marinkovich have filed a patent for animal models and screening methods for psoriasis (PCT/US2017/033609).

Submitted: September 11, 2017 Accepted: November 28, 2017 Published: January 11, 2018

Reference information: JCI Insight. 2018;3(1):e97179. https:// doi.org/10.1172/jci.insight.97179.
Inflammation is critical to atherogenesis. Psoriasis is a chronic inflammatory skin disease that accelerates atherosclerosis in humans and provides a compelling model to understand potential pathways linking these diseases. A murine model capturing the vascular and metabolic diseases in psoriasis would accelerate our understanding and provide a platform to test emerging therapies. We aimed to characterize a new murine model of skin inflammation (Rac1V12) from a cardiovascular standpoint to identify novel atherosclerotic signaling pathways modulated in chronic skin inflammation. The RacV12 psoriasis mouse resembled the human disease state, including presence of systemic inflammation, dyslipidemia, and cardiometabolic dysfunction. Psoriasis macrophages had a proatherosclerotic phenotype with increased lipid uptake and foam cell formation, and also showed a 6 -fold increase in cholesterol crystal formation. We generated a triple-genetic K14-RacV12 ${ }^{-/+} / \mathrm{Srb1}^{-/-} / \mathrm{ApoER} 61^{\mathrm{H} / \mathrm{H}}$ mouse and confirmed psoriasis accelerates atherogenesis ( 7fold increase). Finally, we noted a $60 \%$ reduction in superoxide dismutase 2 (SOD2) expression in human psoriasis macrophages. When SOD2 activity was restored in macrophages, their proatherogenic phenotype reversed. We demonstrate that the K14-RacV12 murine model captures the cardiometabolic dysfunction and accelerates vascular disease observed in chronic inflammation and that skin inflammation induces a proatherosclerotic macrophage phenotype with impaired SOD2 function, which associated with accelerated atherogenesis.

\section{Introduction}

Psoriasis is a chronic inflammatory skin disorder affecting $2 \%-4 \%$ of the population (1) and is associated with an increased risk of atherosclerotic cardiovascular events in humans (2), suggesting shared mechanisms between psoriasis and the presence of cardiovascular disease (CVD). However, mechanistic data linking these 2 disease states are lacking. Given the association with early cardiovascular events, psoriasis provides a reliable model to understand the implications of chronic inflammation on metabolic and CVD (3). Furthermore, utilization of a murine model that recapitulates the human cardiometabolic dysfunction would advance mechanistic understanding but is currently lacking.

Several psoriasis mouse models have been described in the past that have provided critical advancements in our understanding of the cardiovascular effects of chronic systemic low-grade inflammation (4-6). The $K C$-Tie 2 mouse has provided important information on how inflammation in the skin can drive 
inflammation in the vasculature (7) and increase thrombosis (8). Recently, Winge et al. (9) developed a potentially novel psoriasis mouse model, overexpressing a constitutively active mutant ( $\left.\operatorname{Rac} 1^{\mathrm{G} 12 \mathrm{~V}}\right)$ of the GTPase Ras-related C3 botulinum toxin substrate 1 (Rac1), in keratinocytes specifically (K14-Rac1V12), resulting in a comparable pathophysiology between human and murine psoriasis spontaneously manifesting as early as 7 days after birth. This model recapitulated chronic skin inflammation, systemic expression of inflammatory markers, and a psoriasis-associated comorbidity: arthritis.

Macrophages are one of the cellular hallmarks of atherogenesis, and atherosclerosis mouse models have provided important signaling pathways and therapeutic targets $(10,11)$. Psoriasis patients have dense macrophages in psoriatic skin plaques (12) and experience CVD earlier than nonpsoriasis counterparts $(2,13)$. Prior studies involving primary or immortalized macrophages treated with cytokines or psoriasis patient serum demonstrate how a proinflammatory milieu augments macrophage atherogenic functions, but how this occurs is poorly understood $(14,15)$.

A role for manganese in psoriasis has been suggested in 1922 (16) and may be in part mediated by superoxide dismutase 2 (SOD2), a manganese-dependent enzyme. SOD2 is essential for detoxifying cells from reactive oxygen species (ROS) and has been linked to psoriasis (17) and CVD (18), but it has not been characterized in psoriatic macrophages.

Given the recent demonstration of the $\mathrm{K} 14-\mathrm{Rac} 1 \mathrm{~V} 12^{-/+}$psoriasis mouse model recapitulating the psoriasis phenotype (9), including cutaneous monocyte infiltration, our goals of this study were to: i) characterize the model for cardiometabolic disease and signs of atherosclerosis; ii) characterize mouse $\mathrm{K} 14-\mathrm{Rac} 1 \mathrm{~V} 12^{-/+}$-derived macrophages in comparison with human peripheral blood mononuclear cell-derived (PBMC- derived) macrophages from psoriasis patients and to characterize SOD2 in macrophages; and iii) cross the K14-Rac1V12-/+ mouse with the atherosclerosis prone $S r b 1^{-/-} / A p o E R 61^{H / H}$ mouse model to understand if psoriasis-associated systemic inflammation accelerates atherosclerosis development compared with mice lacking the keratinocyte Rac1V12 mutation. Our results provide strong evidence that the K14-Rac1V12-/+ mouse resembles human psoriatic cardiometabolic disease and that the presence of chronic, low-grade inflammation modulates key enzymes in macrophages that contribute to accelerated cholesterol crystal (CC) formation.

\section{Results}

Skin and immune cell characterization show presence of skin-induced systemic inflammation in K14-Rac1V12-/+ mice. As an initial characterization, we sought to confirm that the $K 14-\mathrm{Rac} 1 \mathrm{~V} 12^{-/+}$mouse model of psoriasis (9) developed psoriasis-like skin lesions and skin-specific expression of K14-Rac1V12-/+ (Supplemental Figure 1, A and B; supplemental material available online with this article; https://doi.org/10.1172/jci.insight.97179DS1). Severity of the psoriasis-like skin lesions was graded through a systematic assessment of skin inflammation (Figure 1A and Supplemental Table 1) to provide correlative data on skin severity and systemic disease.

Levels of circulating immune cells in littermate control (LMC) mice compared with psoriasis mice were examined using flow cytometry (Figure 1B). Circulating B and $\mathrm{T}$ cell populations were decreased

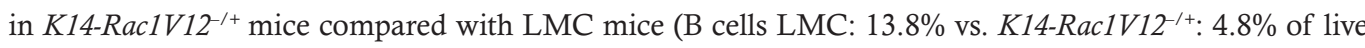
cells; T cells LMC: $11.9 \%$ vs. K14-Rac1V12-/+: $6.5 \%$ of live cells), while neutrophils were increased (LMC: $3.6 \%$ vs. K14-Rac1V12-/+: $13.1 \%$ ). Characterization of the $\mathrm{CD} 11 \mathrm{~b}^{+} / \mathrm{CD} 11 \mathrm{c}^{-}$monocyte population revealed

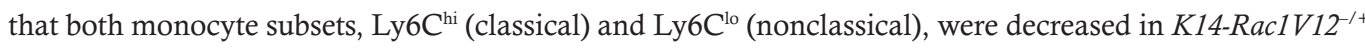
mice (3- and 2-fold decrease, respectively).

Following circulating immune cell characterization, we then measured plasma levels of proinflammatory cytokines that are highly associated to both psoriasis and atherosclerosis (Figure 1C). When compared with LMC, plasma of adolescent (up to 10 weeks of age) as well as adult (over 12 weeks of age) K14Rac1V12-/+ mice displayed significantly increased levels of IL17 (adolescent: LMC: $6.7 \mathrm{pg} / \mathrm{ml}$ vs. K14-

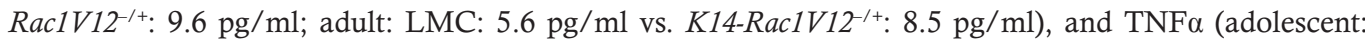

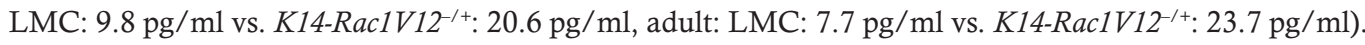
Interestingly, IL6, CXCL1, and IL1 $\beta$ levels were higher in $K 14-R a c 1 V 12^{-/+}$mice; however, their levels only reached statistical significance at adulthood. Furthermore, we analyzed aortic lymph nodes, spleen, and $\mathrm{BM}$ in terms of their immune cell composition to further substantiate the presence of systemic inflammation in $\mathrm{K} 14-\mathrm{Rac}_{\mathrm{V} 12^{-/+}}$mice (Supplemental Figure 1, C-F).

Lastly, transmission electron microscopy (TEM) analysis of mouse aortas revealed the presence of several cell pockets in the subendothelial space that were not present in the aortas of LMC mice (Figure 1D). We delved further into this finding by characterizing the cell populations within the aorta using 
flow cytometry. We did not detect changes in the amount of $\mathrm{CD} 45^{+}$cells within the aortas (Figure $1 \mathrm{E}$ ). We observed an increase in neutrophils by 3.5 -fold, resident DCs by 2.5 -fold and macrophages by 1.6 fold. On the contrary, a significant decrease was observed in conventional DCs by 1.4 -fold $(P<0.01)$. Interestingly, on performing regression analyses, we found that the aortic macrophage content directly correlated with psoriasis severity in $\mathrm{K} 14-\mathrm{Rac} 1 \mathrm{~V} 12^{-/+}$mice (Tables 1 and 2). Due to the observed increased frequency of myeloid cells within the vessel wall, we analyzed the levels of 2 chemoattractants known to be involved in atherogenesis (19) - monocyte chemoattractant protein 1 (MCP-1) and RANTES - and found MCP-1 was significantly upregulated in K14-Rac1V12-/+ mice (LMC, $32.9 \mathrm{pg} / \mathrm{ml}$, vs. K14Rac1V12-/+, $68.1 \mathrm{pg} / \mathrm{ml}$ ), while RANTES was not different (Figure 1F).

Psoriasis-induced shift in lipid profile and dysfunctional HDL. We characterized the lipid profile and HDL function, and we performed metabolic phenotyping. We first used the plasma of fasted mice for fast liquid protein chromatography (FPLC) analysis to determine the overall lipid profile. We found decreased HDL levels and slightly increased VLDL levels (Supplemental Figure 2A). However, LDL levels were significantly decreased in psoriatic mice. Analysis of the plasma lipid showed a decrease in total cholesterol, cholesterol esters, and phospholipids (Supplemental Figure 2B). We also measured serum cholesterol efflux capacity, which is the ability of HDL to accept lipids for reverse cholesterol transport; this has been shown to be impaired in psoriasis patients (20) and accelerate atherosclerosis in humans (21). We found HDL efflux to be significantly decreased by $20 \%$ in the $\mathrm{K} 14-\mathrm{Rac}_{\mathrm{V} 12^{-/+}}$mice (Supplemental Figure 2C).

Metabolic characterization of the $\mathrm{K}_{14-\mathrm{Racl}} \mathrm{V}^{-/+}$mice compared with their LMC showed a 30\% reduced weight with comparable feeding rates, indicating an increased food intake per mouse weight. This suggests heightened metabolic activity of these mice, but it was not statistically significant (Supplemental Figure 2, D-F).

Proatherosclerotic cross-talk between psoriatic macrophages and lipids. We isolated BM-derived macrophages (BMDM) from LMC and K14-Rac1V12-/+ mice to determine if skin-induced inflammation can alter macrophage function. As an initial step, we performed Western blot analysis on BMDMs to prove that the expression of keratinocyte-specific Rac1V12, and its Myc-tag, were not cross contaminating during breeding, clearly showing that K14-Rac1V12 is exclusively expressed in the epidermis of K14-Rac1V12 mice only (Supplemental Figure 1B). No differences in overall Rac1 nor Myc (endogenous and tag) expression could be found comparing LMC and K14-Rac1V12-/+ BMDM, demonstrating that observed effects are due to keratinocyte-specific overexpression of constitutively active Rac1V12 only (Figure 2A).

Differentiation of mouse BM cells to BMDMs (Figure 2B), as well as human PBMC to human monocyte-derived macrophages (HMDMs) (Supplemental Figure 4A), revealed significantly increased precursor-to-macrophage differentiation in both K14-Rac1V12-/+ BMDMs and psoriatic HMDMs, most likely due to differences in adhesion, differentiation, and proliferation. Multivariable regression analysis demonstrated that BMDM differentiation rate is directly correlated with determined psoriasis severity in $K 14$ Rac1V12-/+ mice (Table 2). Moreover, quantitative PCR (qPCR) of BMDMs and HMDMs for genes known to be expressed in polarized, inflamed, and classical macrophages revealed alteration of genes involved in inflammatory and atherosclerotic processes. However, classic genes used in the past to classify M1 macrophages (e.g., TNF $\alpha$, IL1 $\beta$ ) were not altered in K14-Rac1V12-/+ BMDMs but showed alteration in human psoriatic macrophages (Figure 2C and Supplemental Figure 4B). Phenotypically, scanning electron microscopy analysis revealed that nonpsoriatic human and mouse macrophages displayed an elongated phenotype, whereas psoriatic human and mouse macrophages were round and flat (Figure 2, D and E, and Supplemental Figure 4C), indicative of inflammatory macrophages (22). Immunofluorescence staining showed increased expression of CD68 in psoriatic macrophages, while CD206 expression was decreased for both human and mouse macrophages (Figure $2 \mathrm{~F}$ and Supplemental Figure 4D).

We assessed modified LDL uptake by macrophages (Figure 2, G and H). BMDMs derived from K14Rac1V12 ${ }^{-/+}$mice demonstrated increased uptake of acetylated LDL (AcLDL), as well as oxidized LDL (OxLDL), at 2 and 4 hours of incubation compared with LMC BMDMs. We confirmed these results through flow cytometry analyzing the median fluorescence intensities (MFI) of BMDMs (Supplemental Figure 3A); similar results were observed for HMDMs (Supplemental Figure 4, E and F). Accordingly, qPCR analysis of BMDMs and HMDMs showed significantly increased mRNA levels of LDL and modified LDL receptors like CD36 (1.9-fold and 4-fold, respectively) and LDLR (Figure 2I and Supplemental Figure 4I). Furthermore, we observed a 1.6-fold increase in macrophage foam cell formation (Figure $2 \mathrm{~J}$ ) at baseline without the addition of external lipids, which was consistent even after the additional treatment of AcLDL and OxLDL, confirmed by flow cytometry (Supplemental Figure 3B) and positively correlated with severity (Table 2). Again, these results 
A Severity grade

$\square 1.71 \% 0 \square 27.8 \% 3$

$\square 7.69 \% 1 \square 18.6 \% 4$

$\square 33.3 \% 2 \square 10.2 \% 5$

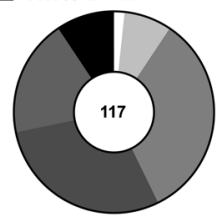

B

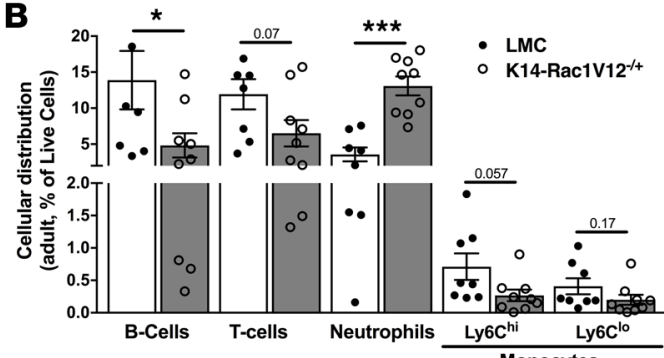

C
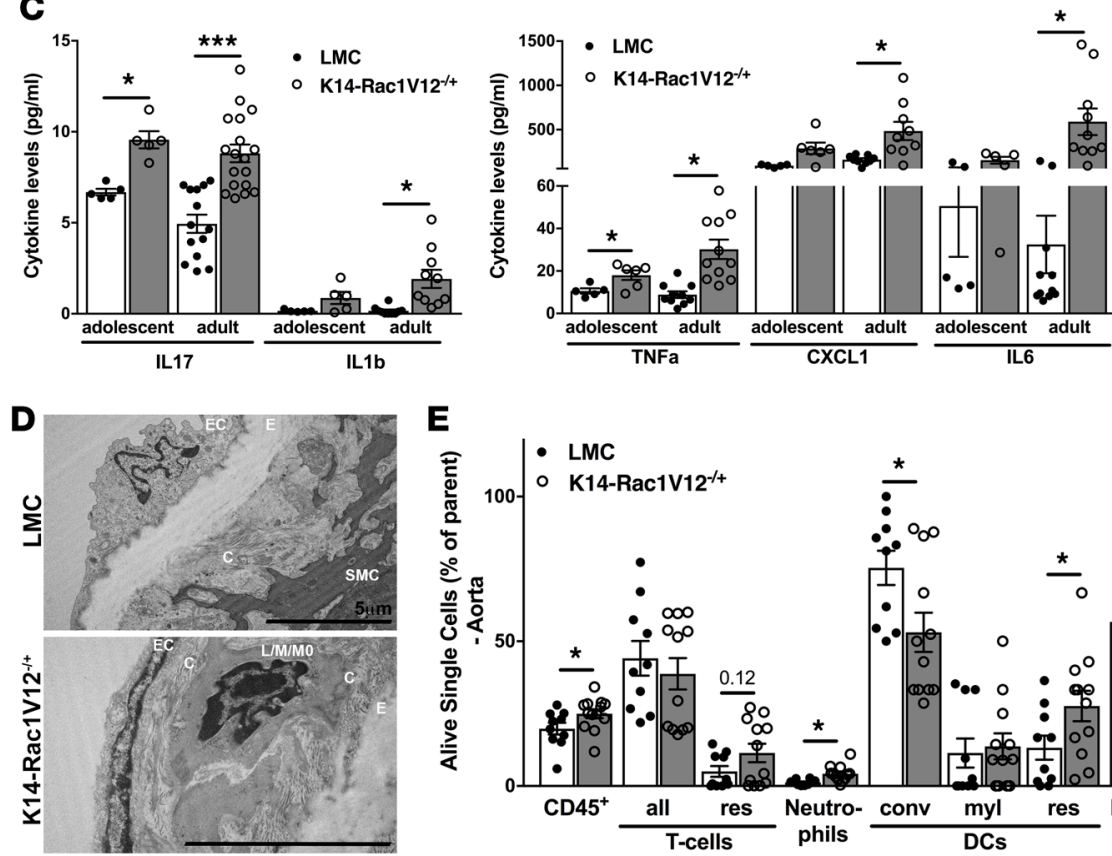

$\mathbf{E}$
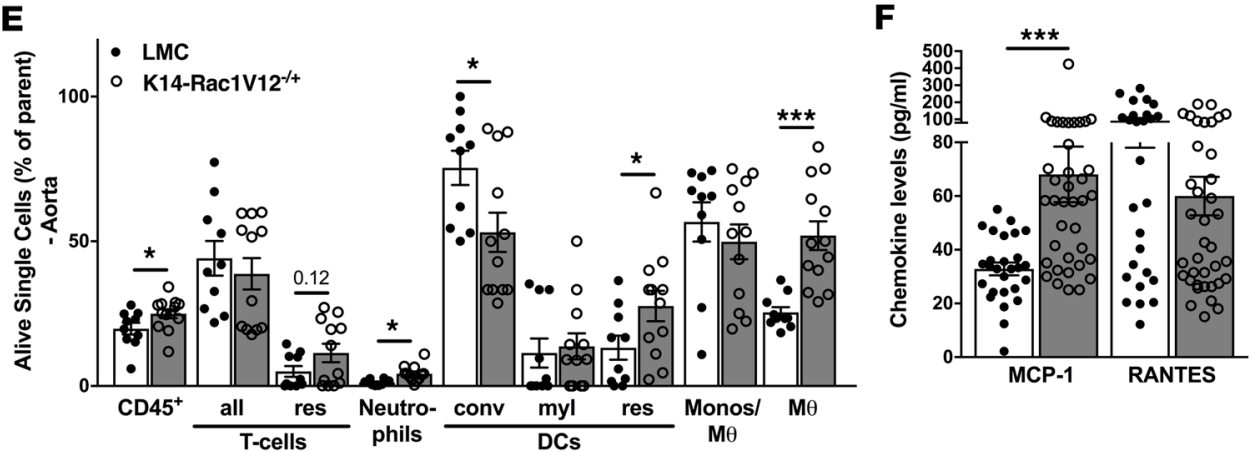

Figure 1. Overexpression of constitutive active Rac1 V12 in keratinocytes induces psoriasis accompanied by systemic chronic inflammation. (A) Severity of psoriasis phenotype based on visible symptoms was determined on a $0-5$ scale. Severity levels of 2 and 3 accounted for the majority of all animals ( $n=$ 117). (B) Blood cell composition differences were determined by flow cytometry. Decreased levels of B cells, T cells, classical (Ly6Chi), and nonclassical (Ly6Clo) monocytes were observed, while the neutrophil population increased $(n=8 / 9)$. (C) Chronic inflammation was detected by measuring plasma cytokine levels in adolescent (younger than 10 weeks) and adult (older than 12 weeks) K14-Rac1V12 ${ }^{-/+}$mice and their LMC. Increased levels of all proinflammatory cytokines were detected $(n>5)$. (D) Transmission electron microscopy of mouse aortas showed infiltration of myeloid cells into the subendothelial space ( $n=3 / 3$ ) and the tunica media, which was confirmed by flow cytometry (E) of Liberase-digested aortas ( $n=10 / 13$ ). Neutrophils, resident (res) DCs and especially macrophages are showing the most significant changes. (F) Plasma from adult LMC and K14-Rac1V12//+ mice were used to determine MCP-1 and RANTES chemokine levels ( $n=27 / 38)$. (Data are expressed as mean \pm SEM, $n=L M C / K 14-R a c 1 V 12^{-/+}$; Mann-Whitney test $\left.P<0.05\right)$ (LMC, littermate control; EC, endothelial cell; E, elastin layer; C, collagen; SMC, smooth muscle cell; L/M/Mø, lymphoid or myeloid cell; MØ, macrophages; conv, conventional; res, resident; myl, myeloid; Monos, monocytes; M0, macrophages; MCP-1 (CCL2), monocyte chemoattractant protein 1. Scale bar: $5 \mu \mathrm{m} .{ }^{*} P<0.05,{ }^{* *} P<0.0005$.

were corroborated in HMDMs (Supplemental Figure 4G). Filipin staining as an indicator for free cholesterol content was determined and showed a significant increase at baseline, while addition of AcLDL resulted in decreased Filipin staining in K14-Rac1V12-/+ BMDMs compared with LMC BMDMs (Supplemental Figure 3C). Additionally, we found that macrophage-cholesterol efflux was similar between LMC and psoriatic mouse BMDMs (Supplemental Figure 3D). HMDMs from psoriasis patients, on the other hand, had impaired macrophage-cholesterol efflux compared with healthy volunteer HMDMs (Supplemental Figure 4H). Using qPCR, we analyzed psoriasis-induced alterations in genes associated with lipid metabolism $(A C A T / S O A T, N C E H)$ and efflux transporters ( $A B C A 1, A B C G 1$ ) (Supplemental Figure 3E and Supplemental 4I). A 50\% downregulation of the $A B C G 1$ gene was found only in HMDMs and a significant increase of $A C A T / S O A T$ was found in both macrophage types, while $N C E H$ was regulated in opposite ways between human and mouse psoriatic macrophages. Subsequently, we performed mouse and human macrophages apoptosis/necrosis experiments (Supplemental Figure 3F and Supplemental Figure 4J). Early apoptotic events were significantly increased in both K14Rac1V12 $2^{-/+}$mouse BMDMs and human psoriasis HMDMs when compared with LMCs and healthy volunteers, respectively, not involving activation of NLRP1 or NLRP3 inflammasome (Supplemental Figure 3G). 
Table 1. Description of study groups for different mouse models

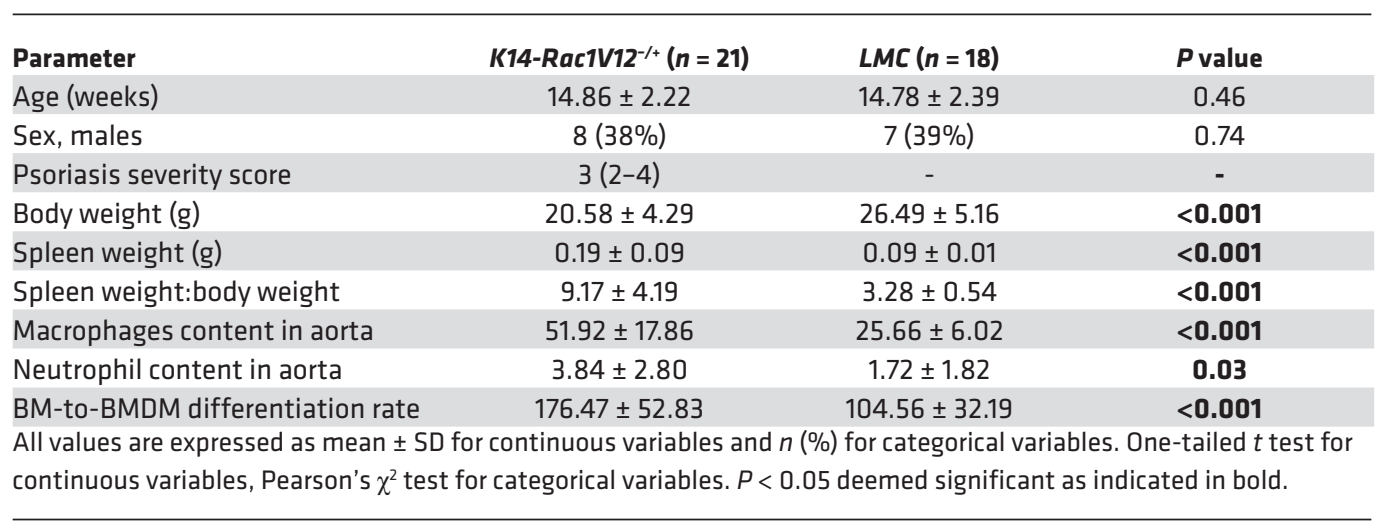

CCs were seen in macrophage foam cells using polarized light microscopy (Figure 2, $\mathrm{K}$ and $\mathrm{L}$ ). We observed a formation of CCs within psoriatic mouse BMDMs and HMDMs that was accelerated with the addition of lipids. Nonpsoriatic macrophages displayed minor to no presence of CC formation, even upon lipid introduction.

Psoriasis accelerates atherosclerosis development. K14-Rac1V12-/+ mice were successfully crossed with Srb1I-/ApoeR61 ${ }^{H / H}$ mice to create an atherosclerosis-prone psoriatic mouse (Supplemental Figure 5A). These mice displayed mild-to-moderate psoriasis phenotypes, with increasing severity when being fed a high fat diet (HFD) (Figure 3A), a significantly lower body weight (Supplemental Figure 5B), and an increased spleen/body weight ratio (Supplemental Figure 5C) after being on a HFD for 2 weeks. Furthermore, 2 weeks of HFD resulted in increased presence of neutrophils in peripheral blood in $S r b 1^{-/-} / A_{p o e R 61^{H / H} /}$

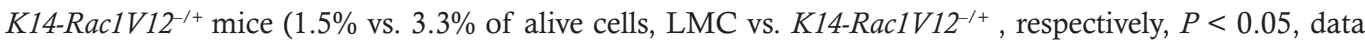
not shown). Lipid analysis revealed no differences in total triglycerides, total free cholesterol and esterified cholesterol (Table 3). Additionally, after a HFD, the triple genetic mice displayed an increased presence of $\mathrm{CD} 45^{+}$cells and $\mathrm{T}$ cells in aortic lymph nodes; assessment of the spleen revealed a decreased presence of monocytes, while macrophages were increased (Supplemental Figure 5, D and E).

En face analysis of whole mouse aortas revealed an increase in atherosclerotic plaque formation in $\mathrm{Srb1}^{-/-} /$ ApoeR61 $1^{H / H} / \mathrm{K} 14-\mathrm{Rac} 1 \mathrm{~V} 12^{-/+}$mice as compared with their age- and sex-matched LMC $(6.8 \%$ vs. $1.8 \%$ plaque area of whole aorta, respectively) (Figure 3B). This increase in atherosclerotic plaque formation was confirmed by Oil Red O staining in aortic root sections (Figure 3C, $0.04 \mathrm{~mm}^{2}$ Srb1 $1^{-/-} /$ApoeR61 $1^{H / H} / \mathrm{LMC}$ vs. $0.11 \mathrm{~mm}^{2}$ $\mathrm{Srb1}^{-/-} /$ApoeR $\left.61^{H / H} / \mathrm{K} 14-R a c 1 V 12^{-/+}\right)$. Furthermore, severity of atherosclerotic lesion formation correlated with severity of the psoriasis phenotype. Atherosclerotic plaque of Srb1 $1^{-/-} / A p o e R 61^{H / H} / \mathrm{K} 14-R a c 1 V 12^{-/+}$were characterized to be macrophage rich with no signs of calcification (Figure 3, D and E). No differences in macrophage or smooth muscle cell content were observed. However, an increased presence of CCs and necrotic core area was seen in Srb1 $1^{-/-} /$ApoeR61 $1^{H / H} / K 14-R a c 1 V 12^{-/+}$mice, even after normalization to overall atherosclerotic lesion size (Figure 3, D and E). Staining of collagen using Sirius Red displayed no significant differences in overall collagen content (bright field red) or in the thickness of collagen fibers (Sirius Red staining in polarized light: green/ yellow indicates thin fibers, orange/red indicates thick collagen fibers) (Figure 3F).

SOD2 is a regulator of psoriasis-induced proatherosclerotic macrophage dysfunction. As SOD2 is essential for macrophage function and counteracting oxidative stress (23), we speculated that SOD2 function might be impaired in psoriatic macrophages, resulting in enhanced presence of mitochondrial ROS. Mitochondrial ROS were significantly increased by 2-fold in human PBMCs and increased by 1.8 -fold in psoriatic mouse BM cells, the precursors to our derived macrophages, suggesting a possible modulation of SOD2 in macrophage function and development (Figure 4A and Supplemental Figure 6A). qPCR analysis of human and mouse macrophages (Figure 4B and Supplemental Figure 6B) for mitochondria-related genes revealed SOD2 to be commonly downregulated in both species of psoriatic macrophages. The additional downregulation of Sirt3 (Sirtuin 3) in K14-Rac1V12-/+ BMDM, which is a regulator of SOD2 activity (24), provided further evidence for psoriasis-induced alteration in macrophage SOD2 expression and activation. Flow cytometry-based analysis of mitochondrial ROS in differentiated macrophages revealed increased presence of mitochondrial ROS 
Table 2. Multivariable regressions

Psoriasis score and body weight in $\mathrm{K} 14-\mathrm{Rac} 1 \mathrm{~V} 12^{-/+}$

\section{Model}

Unadjusted

Adjusted for age

Adjusted for age and sex

Psoriasis score and spleen weight in K14-Rac1V12-/+

\section{Model}

Unadjusted

Adjusted for age

Adjusted for age and sex

Psoriasis score and spleen weight:body weight in K14-Rac1V12-/

\section{Model}

Unadjusted

Adjusted for age

Adjusted for age and sex

Psoriasis score and aortic macrophages in K14-Rac1V12-1

\section{Model}

Unadjusted

Adjusted for age

Adjusted for age and sex

Psoriasis score and K14-Rac1V12-/+ BMDM differentiation rate

Model

Unadjusted

Adjusted for age

Adjusted for age and sex

Psoriasis score and K14-Rac1V12-/+ BMDM foam cell formation after AcLDL treatment

Model

Unadjusted

Adjusted for age

Adjusted for age and sex

Psoriasis score and K14-Rac1V12-/+BMDM mitochondrial ROS

Model

Unadjusted

Adjusted for age

Adjusted for age and sex

Data represented as standardized $\beta$ coefficient, $P<0.05$.
K14-Rac1V12 $^{-/+}(n=21)$

$-0.48(0.03)$

$-0.49(0.02)$

$-0.63(0.047)$

K14-Rac1V12 $^{-/+}(n=21)$

$0.75(<0.001)$

$0.75(<0.001)$

$0.73(<0.001)$

$K_{14-R a c 1 V 12-/+}(n=16)$

$0.90(<0.001)$

$0.87(<0.001)$

$0.76(<0.001)$

K14-Rac1V12-/+ $(n=13)$

$0.86(<0.001)$

$0.91(0.001)$

$0.72(0.011)$

K14-Rac1V12 $^{-/+}(n=21)$

$0.68(0.001)$

$0.67(0.001)$

$0.64(0.003)$

in psoriatic macrophages (Figure 4C and Supplemental Figure 6D). Since SOD2 is a manganese-dependent superoxide dismutase, we sought to differentiate precursor cells in the presence of manganese, which resulted in decreased macrophage numbers after the differentiation process in psoriatic human and mouse macrophages (Figure 4D and Supplemental Figure 6C). Manganese-differentiated psoriatic mouse macrophages displayed alterations in their genetic profile compared with those without the addition of manganese, especially in lipid uptake and efflux-regulating genes (Figure 4E). To determine actual SOD2 activity instead of mitochondrial ROS, a SOD2 activity indicator, we performed a SOD2 activity assay in BMDM (Figure 4F) In this assay, SOD2 activity is measured as inhibition rate, which directly correlates with SOD2 activity. We found: i) decreased SOD2 activity in K14-Rac1V12-/+ BMDMs compared with LMC; ii) that differentiation of macrophages with manganese did not significantly affect SOD2 activity; and iii) that the addition of MnTBAP, a SOD2 mimetic, rescued K14-Rac1V12-/+ BMDM SOD2 activity deficiency in manganese-differentiated macrophages, suggesting that activation of residual SOD2 is not sufficient to restore psoriasis-altered SOD2 deficiency. To further confirm that alteration of SOD2 activity impacts mitochondrial ROS presence, microscopy (Figure 4G) and flow cytometry (Figure $4 \mathrm{H}$ ) were used. In both assays, either differentiation of K14-Rac1V12-/+ BMDMs in the presence of manganese or the addition of MnTBAP were sufficient to substantially decrease the psoriasis-induced increase in mitochondrial ROS to LMC levels. 

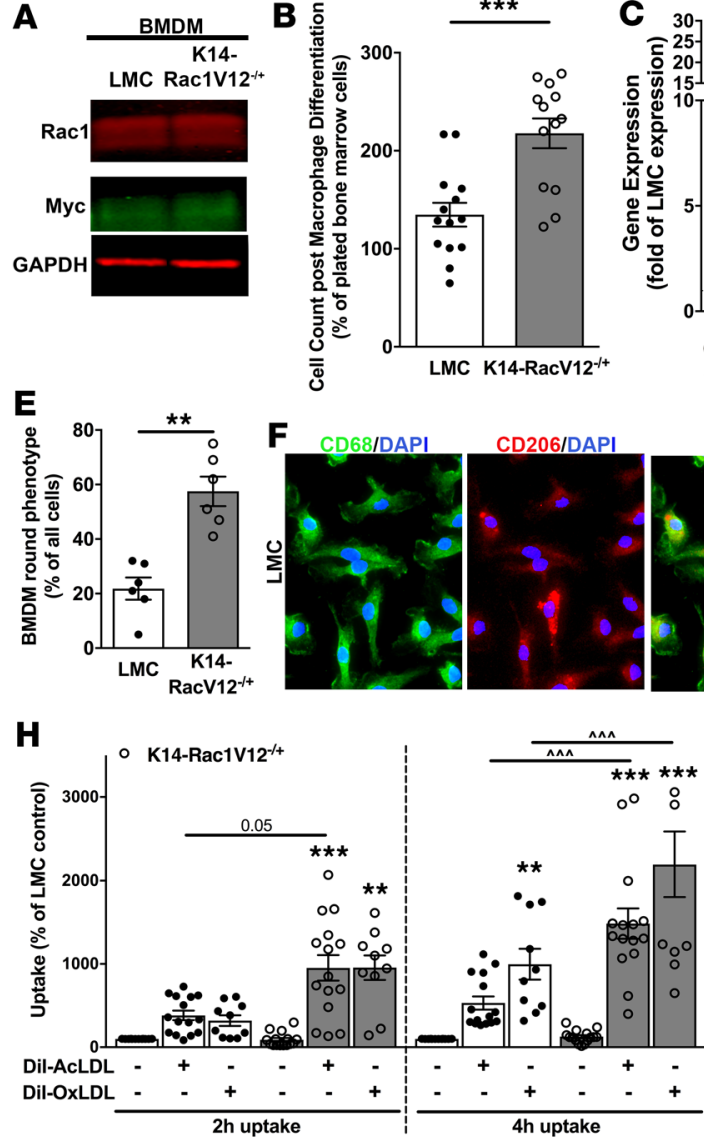

$\mathbf{K}$
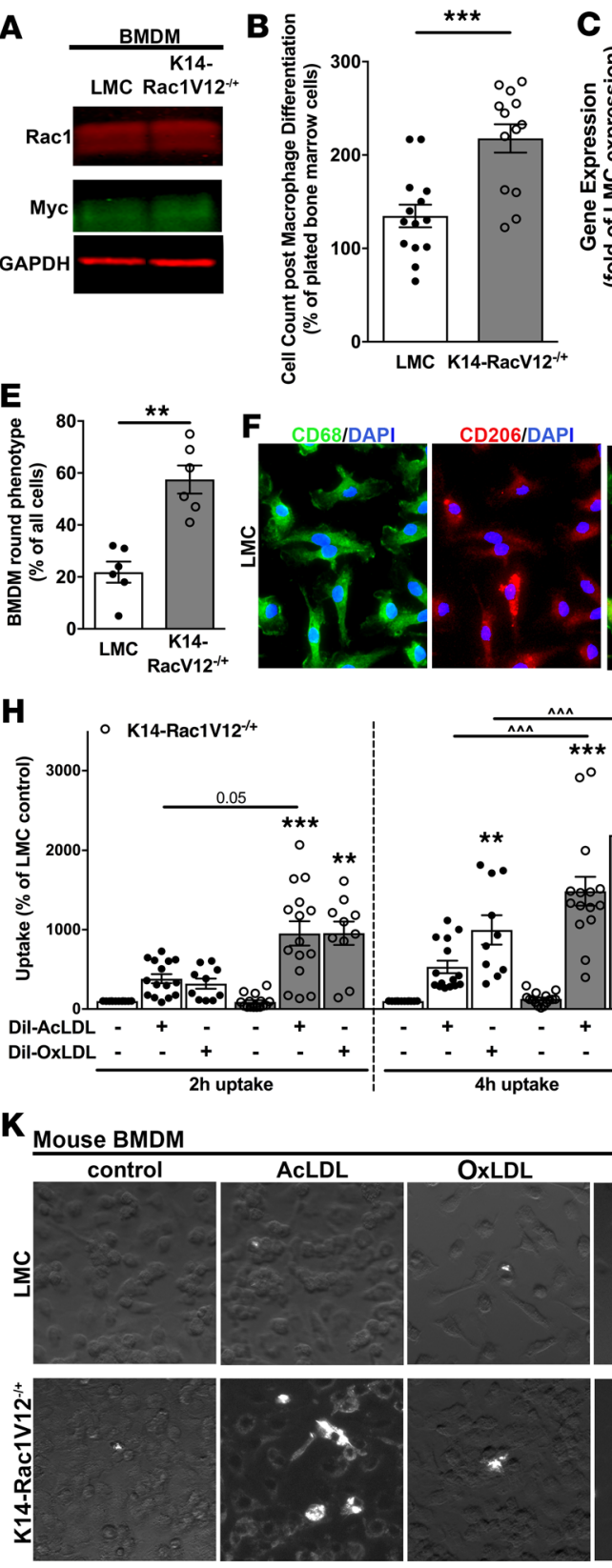
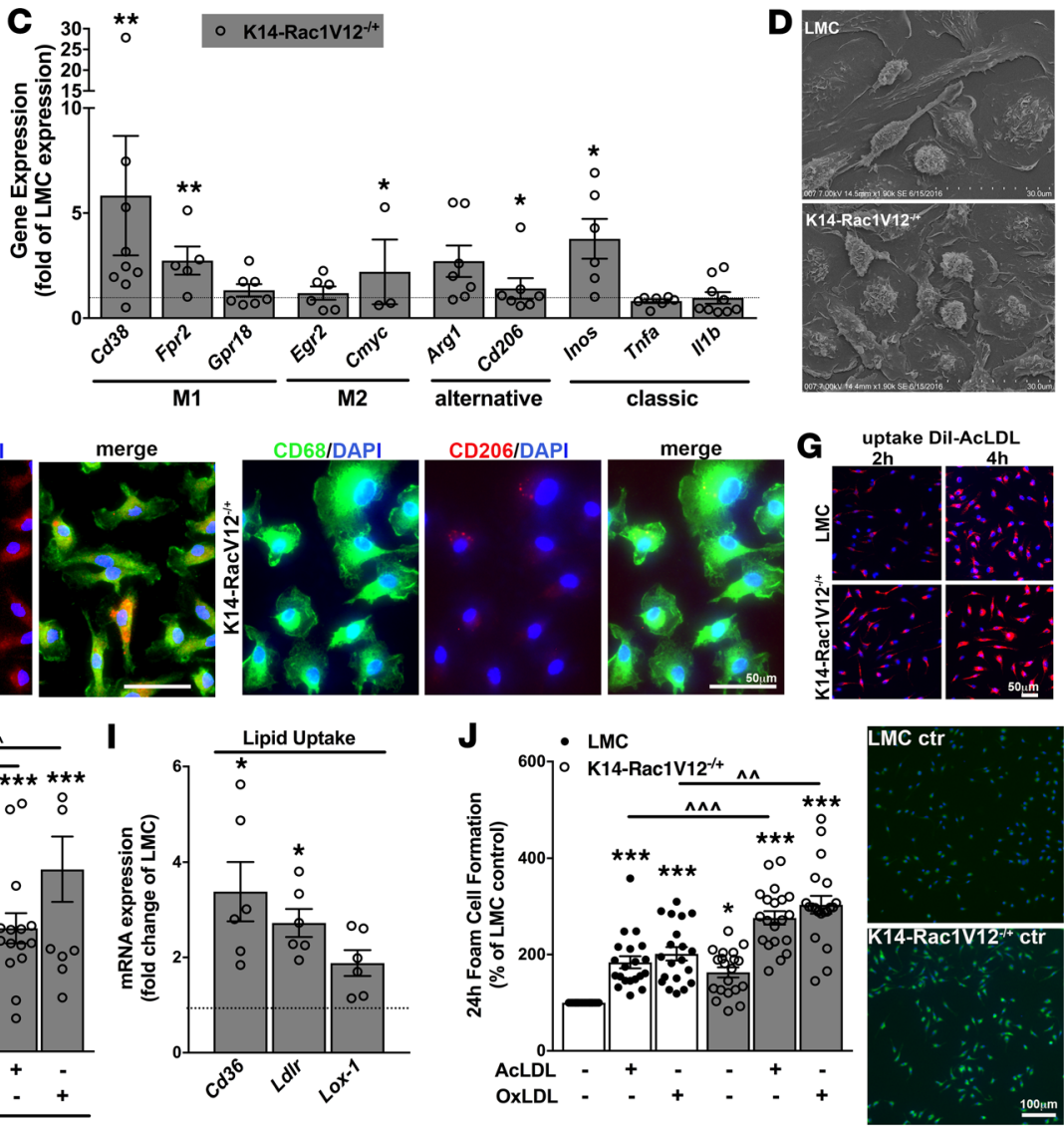

L Human HMDM
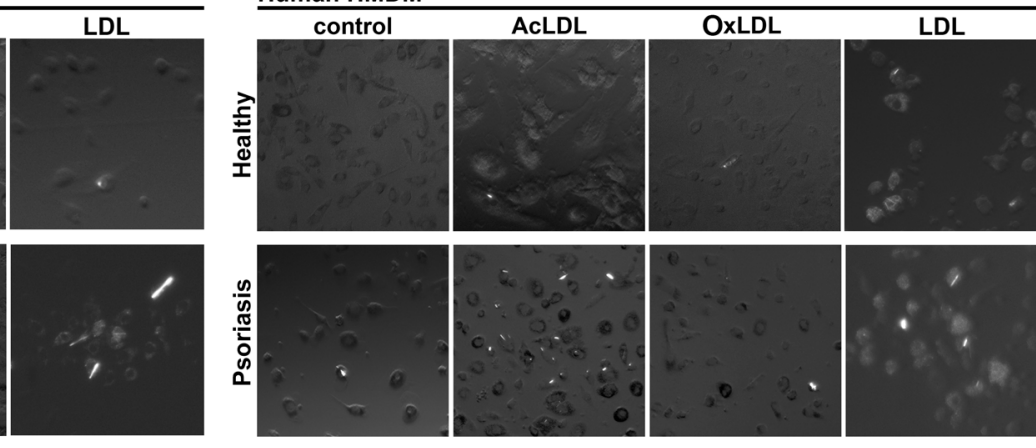

Figure 2. Psoriatic mouse macrophages display proatherosclerotic characteristics. (A) BMDM were analyzed for the presence of Rac1 and the attached Myc tag. No differences in expression of Rac1 or Myc were detected. (B) Equal amounts of BM cells were plated for differentiation. Cell count after differentiation was determined. Resulting cell counts are increased in psoriatic mice, possibly due to differences in adhesion and proliferation. (C) Gene expression of macrophage polarization was determined using qPCR and is altered in psoriatic BMDMs as compared with LMC BMDMs. (D and E) Scanning electron microscopy analysis of BMDM phenotype with subsequent quantification of cells presenting a round phenotype ( $n=6$, each with 5 fields imaged and quantified). (F) Phenotypically, BMDMs were characterized using scanning electron microscopy and immunofluorescence analysis for CD206 (red) and CD68 (green) (nuclei: DAPI, blue) (representative images of $n=3$ ). ( $\mathbf{G}$ and $\mathbf{H}$ ) Modified lipoprotein uptake by BMDM was determined after treatment with $20 \mu \mathrm{g} / \mathrm{ml}$ Dil-labeled AcLDL or OxLDL for $2 \mathrm{~h}$ and $4 \mathrm{~h}$ of incubation. Five images per condition were taken at equal exposure and MFI of 100 individual cells per image analyzed using ImageJ. (I) Differences in modified LDL receptors between LMC and K14-Rac1V12-/+ BMDM were detected using qPCR. (J) Macrophage foam cell formation upon $24 \mathrm{~h} 50 \mu \mathrm{g} / \mathrm{ml}$ AcLDL or OxLDL treatment was detected after subsequent Bodipy493/503 labeling (green, nuclei: DAPI, blue) and ImageJ analysis as described in $\mathbf{E}$ and $\mathbf{F}$. (K and $\mathbf{L}$ ) Presence of cholesterol crystals was visualized using polarized light microscopy of mouse (K) and human (L) macrophages, displaying increased cholesterol crystal presence under baseline and lipid treated conditions $(50 \mu \mathrm{g} / \mathrm{ml})(B M D M n=6 / 6, \mathrm{HMDM} n=5 / 10)$. (Data are expressed as mean $\pm \mathrm{SEM}$; 2-way ANOVA with Bonferroni correction [H and J] with $P$ $<0.007$; duplicates for $\mathbf{H}$ and J; Mann-Whitney test [B, C, E, and I] with $P<0.05 ; n \geq 6 / 6,{ }^{*} P<0.05,{ }^{* *} P<0.005,{ }^{* *} P<0.0005$ indicates significance to $L M C$ control; ${ }^{\wedge} P<0.05,{ }^{\wedge} P<<0.005,{ }^{\wedge \wedge} P<<0.0005$ indicates significance between indicated groups; scale bars: $50 \mu \mathrm{m}$ [F and $\left.\left.\mathbf{C}\right], 100 \mu \mathrm{m}[\mathrm{J}]\right)(\mathrm{LMC}$, littermate control; AcLDL, acetylated low-density lipoprotein; OxLDL, oxidized LDL) 
A

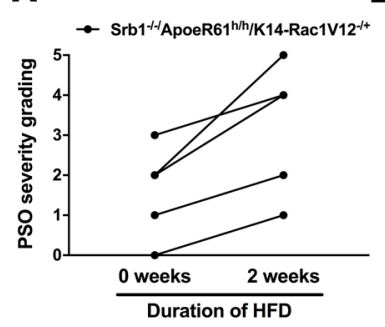

B

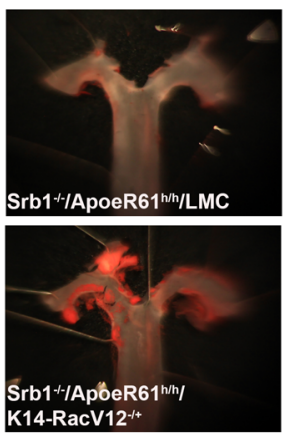

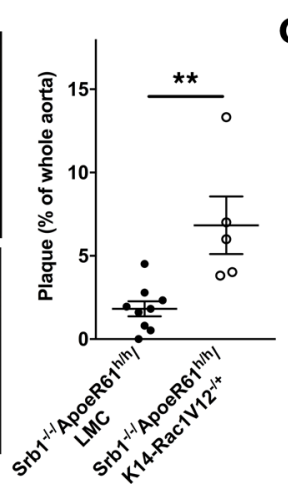
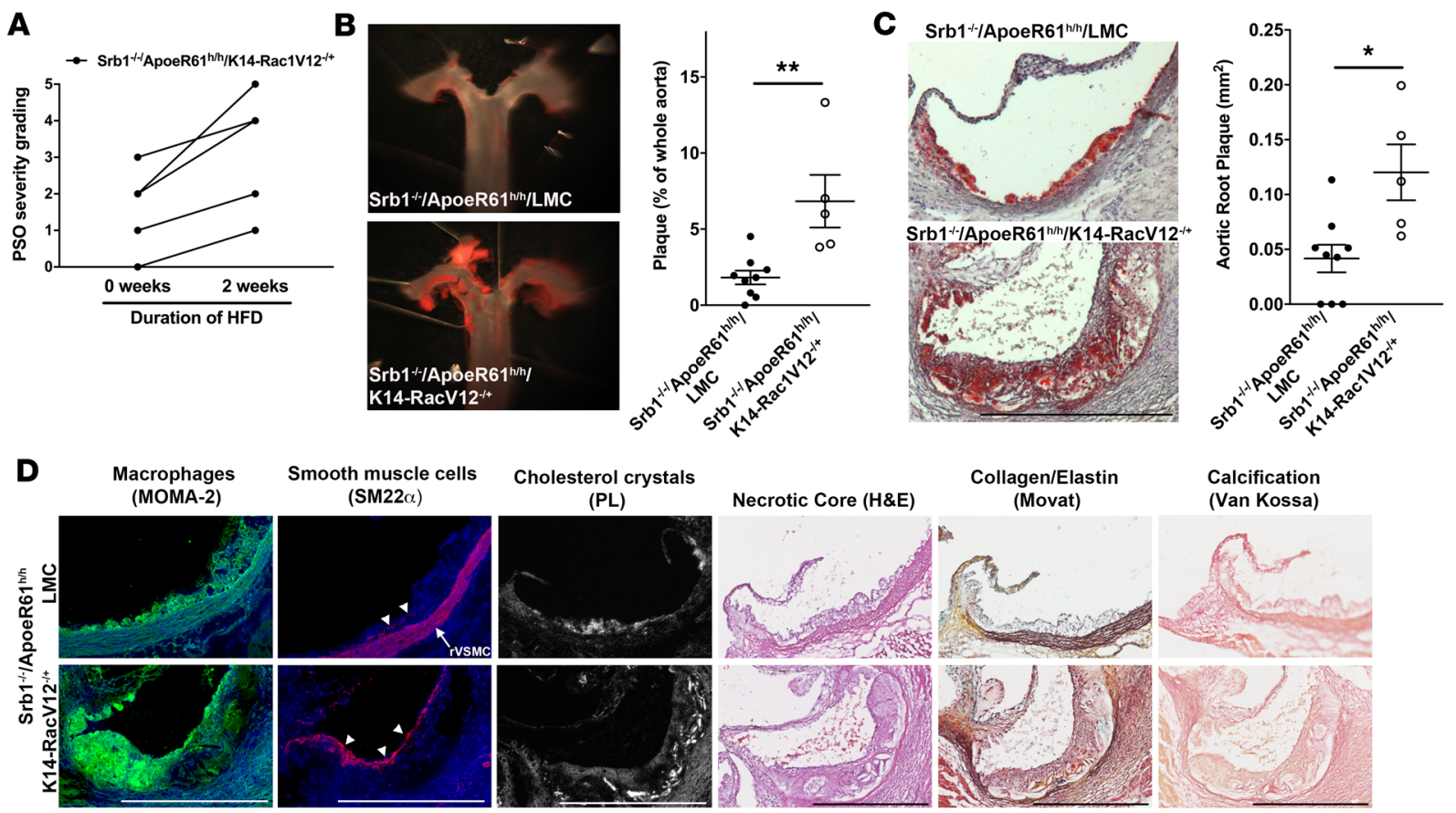

Necrotic Core (H\&E)

Collagen/Elastin (Movat)
Calcification (Van Kossa)
E

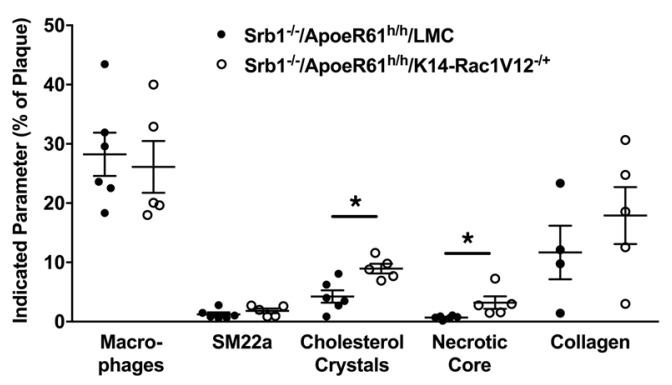

$\mathbf{F}$

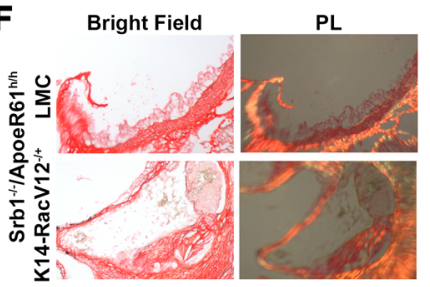

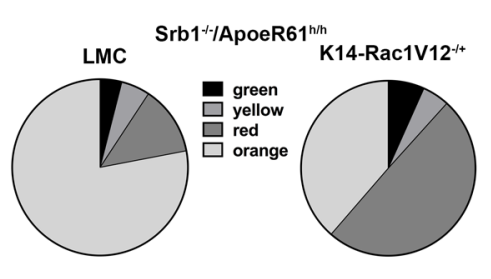

Figure 3. Psoriasis-induced chronic inflammation accelerates atherogenesis. $K 14-R a c 1 V 12^{-/+}$mice were backcrossed into $\mathrm{Srb} 1^{-/-} / \mathrm{Apoe} R 61^{H / H}$ mice and an atherosclerosis study conducted. (A) Psoriasis severity of Srb1 $1^{-1 /} / A p o e R 61^{H / H} / K 14-R a c 1 V 12^{-/+}$mice increased due to HFD for 2 weeks. After 2 weeks of HFD, detection of atherosclerotic plaques in whole aorta (B, images presented of the aortic arch) and aortic root sections (C) using Oil Red 0 staining showed a significant increase in atherosclerosis development in psoriatic mice vs. LMC mice. (D) Macrophage (MOMA-2), smooth muscle cell (SM22 $\alpha$ ) and cholesterol crystal content, necrotic core area, general collagen and elastin (Movat) deposition, and calcification (Van Kossa) in the aortic root of animals presenting plaque formation in $\mathbf{C}$ are shown to compare the anatomy and quality of the developed atherosclerotic lesions. Quantifications (E) were done using ImageJ software and are displayed as percentage of total plaque area. (F) Sirius Red staining was performed and visualized using bright field and polarized light microscopy. Content of different collagen fiber thicknesses was determined using Image). Green/yellow indicates thinner collagen fibers, while orange/ red indicates thick collagen fibers. (Data are expressed as mean \pm SEM, Mann-Whitney test with ${ }^{*} P<0.05,{ }^{* *} P<0.005, n=9 / 4$ for Oil Red 0 staining, $n=$ 6/4 for plaque characterization staining, scale bar: $500 \mu \mathrm{m}$ ) (PL, polarized light; CC, cholesterol crystals; HFD, high-fat diet; PSO, psoriasis; rVSMC, resident vascular smooth muscle cells [indicated by the arrows]. Arrow heads indicate plaque/infiltrated VSMC).

To determine the role of SOD2 in psoriasis-induced proatherosclerotic macrophages, macrophage function experiments were performed in manganese-differentiated BMDMs and HMDMs with and without the addition of MnTBAP. Psoriasis-induced early apoptotic events were significantly reduced to LMC event levels in manganese-differentiated K14-Rac1V12-/+ BMDMs (Figure 5A). Moreover, modified lipid uptake and foam cell formation of $\mathrm{K} 14-\mathrm{Rac} 1 \mathrm{~V} 12^{-/+} \mathrm{BMDMs}$ were both reduced to LMC levels in manganese-differentiated macrophages, with further significant reduction due to the addition of MnTBAP (Figure 5, B and C). Cholesterol efflux of psoriatic BMDMs was not significantly altered by the different treatment conditions (Figure 5D). Finally, we assessed if SOD2 activity was related to CC formation. We observed a significantly lower presence of CCs in manganese-differentiated K14-Rac1V12-/+ BMDMs treated with MnTBAP (Figure 5, E and F). In addition, we observed a reduction to LMC levels in lipid-induced CC formation in manganese-differentiated psoriatic BMDMs with MnTBAP treatment. Similar results were obtained using human psoriatic macrophages under the given conditions (Supplemental Figure 6, E-G). 
Table 3. Summary of lipid analysis of atherosclerosis study mice

\begin{tabular}{|c|c|c|}
\hline & Srb1 $1^{-/-} /$ApoER61 $1^{H / H} /$ LMC & Srb1 $1^{-/-} /$ApoER61 $^{H / H} /$ K14-Rac1V12 $^{-/+}$ \\
\hline Total free cholesterol (mg/dl) & $185.5 \pm 6.3$ & $186.2 \pm 9.2$ \\
\hline Total esterified cholesterol (mg/dl) & $237.7 \pm 7.8$ & $267.5 \pm 56.6$ \\
\hline Total triglycerides (mg/dl) & $157.4 \pm 12.9$ & $160.3 \pm 15.1$ \\
\hline
\end{tabular}

Data presented as mean \pm SEM.

\section{Discussion}

Multiple studies demonstrate an increased risk of cardiometabolic dysfunction and atherosclerotic CVD in human psoriasis $(2,13)$. In this study, we determined that the recently published Rac1V12 murine model of psoriasis (9) recapitulated several cardiometabolic dysfunction features found in human psoriasis. Furthermore, we found that: i) psoriatic macrophages in human and mice are proatherosclerotic and display increased lipid uptake, foam cell formation, and CC formation; ii) psoriasis induces SOD2 dysfunction in psoriatic mouse and human macrophages, and iii) replenishment of SOD2 reverses the proatherosclerotic macrophage phenotype. Additionally, we successfully generated a triple genetic atherosclerosis-prone psoriasis mouse model $\left(\mathrm{Srb1}^{-I_{-}} / \mathrm{ApoeR} 61^{H / H} / \mathrm{K} 14-\mathrm{RaCl}_{\mathrm{V} 12^{-/+}}\right)$and demonstrated, for the first time to our knowledge, that a psoriatic phenotype displays an earlier onset of atherosclerosis and that an epidermal-intrinsic defect can drive systemic CVD progression. Taken together, these results highlight the importance of chronic inflammation in driving macrophage dysfunction and ultimately atherosclerosis development and progression.

In patients with psoriasis, vascular inflammation is observed using 18-fludeoxyglucose PET/CT (FDG-PET/CT) signal in large vessels, namely the aorta - the signals of which have a dose-response relationship to psoriasis severity (25). The FDG-PET/CT signal is believed to be derived from infiltrative macrophages residing within the vessel wall (26). A first indicator that a psoriatic mouse model expresses signs of vascular inflammation has been reported by Wang et al. (7) using histological staining methods to determine white blood cell infiltration in the aortic root of $K C$-Tie 2 mice on a chow diet. In our study, we visualized cell infiltration within the vessel wall using TEM and identified the subpopulations extensively using flow cytometry analysis. While several cell-type populations shift within the K14-Rac1V12-/+ vessel wall, the increased presence of macrophages, strongly believed to drive FDG-uptake in human psoriasis, is indicating a proatherosclerotic preconditioning of the vessel wall. Additionally, we were able to demonstrate a positive correlation of macrophage content with psoriasis severity (Tables 1 and 2).

Serum HDL efflux is inversely correlated with noncalcified burden (21) in the coronaries of psoriasis patients, accompanied by profound changes in the lipid profile (27) and prospective cardiovascular events in humans (28). Analysis of psoriatic mouse HDL revealed its decreased capability to promote reverse cholesterol transport, similar to our psoriasis patients, further supporting the proatherosclerotic phenotype of the K14-Rac1V12-/+ murine psoriasis model.

Atherosclerosis and psoriasis are chronic inflammatory diseases accompanied by changes in blood cell composition and cytokine levels. Our group has shown in the past that psoriasis has increased neutrophil cell counts, which show a direct positive association with psoriasis severity (25). The role of monocytes and their subsets in human psoriasis was highlighted in a recent publication (8), which is in accordance to our mouse data presented in this study. These findings are in contrast with data demonstrating that $K C$-Tie 2 mice display increased levels of circulating and splenic $C D 11 b^{+}$Ly $6 C^{\text {hi }}$ proinflammatory monocyte subsets (7). Although we saw a decrease in classical/inflammatory monocyte subset levels, we postulate that significant amounts of monocytes are recruited to target organ tissues (e.g., aorta) and subsequently differentiated to macrophages. Ly6 $\mathrm{C}^{\text {hi }}$-monocyte-derived macrophages (Ly6C ${ }^{\text {hi }}$-MDMs) reside long-term within tissue, suggesting a link between inflammation severity, duration, and Ly6 $\mathrm{C}^{\text {hi }}$ monocyte-derived macrophage content (29). Furthermore, the importance of inflammatory Ly6 $\mathrm{C}^{\text {hi }}$ monocytes and their differentiation into M2 macrophages in atherosclerosis regression has been published very recently utilizing a mouse plaque transplant model (30), indicating that the observed decrease in inflammatory Ly6 $\mathrm{C}^{\mathrm{hi}}$ monocytes impairs plaque regression and probably sup- 
A

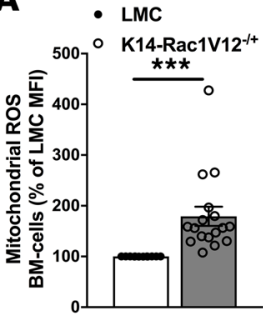

B

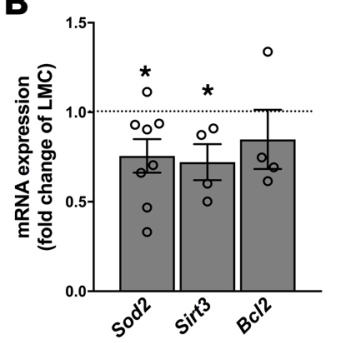

C

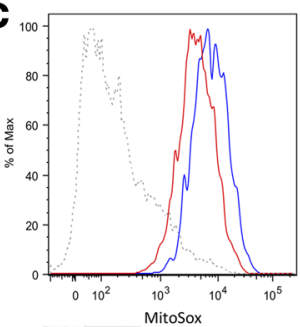

9 Unstained BMDM 80.1 Stained LMC BMD 82.3 Stained K14-Rac1V12
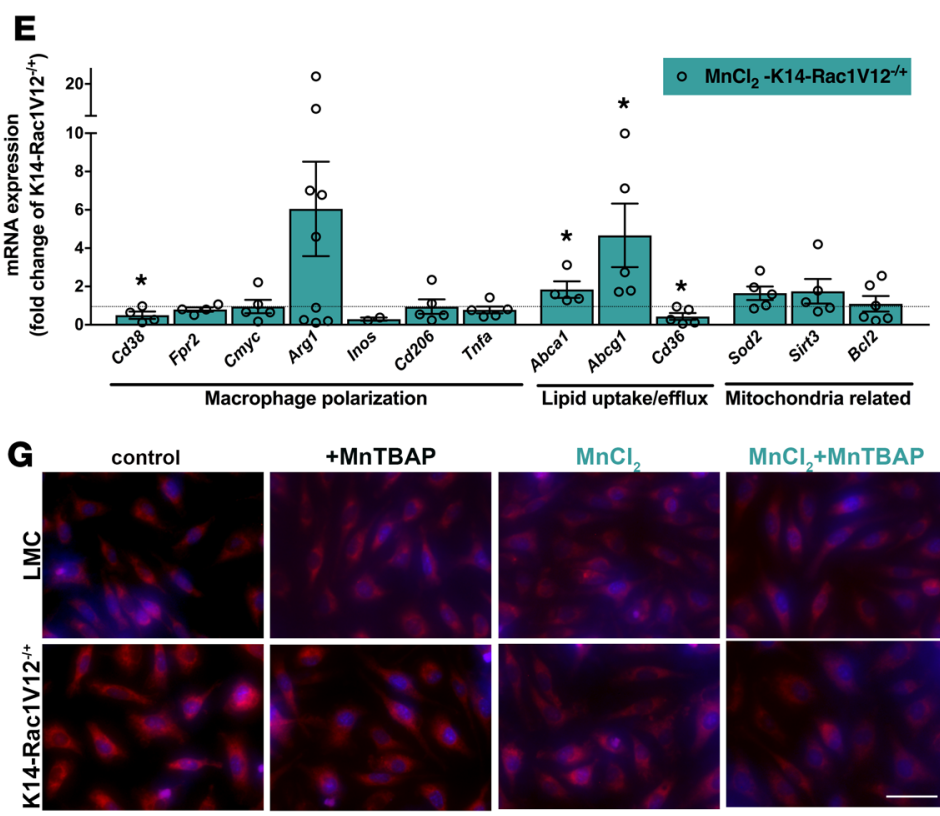

+MnTBAP
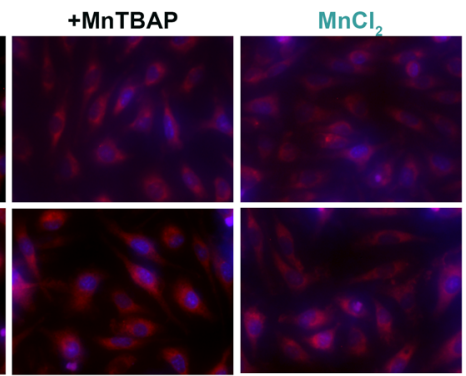

$\mathrm{MnCl}+\mathrm{MnTBAP}$

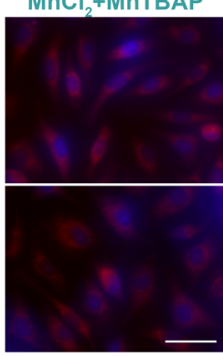

H

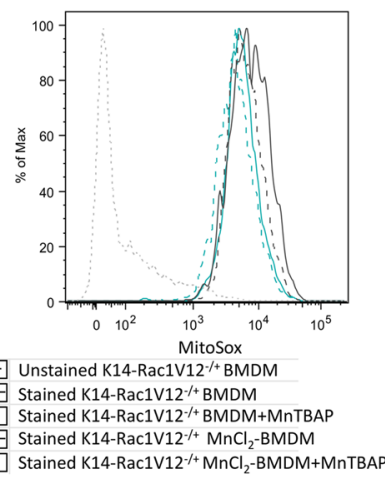

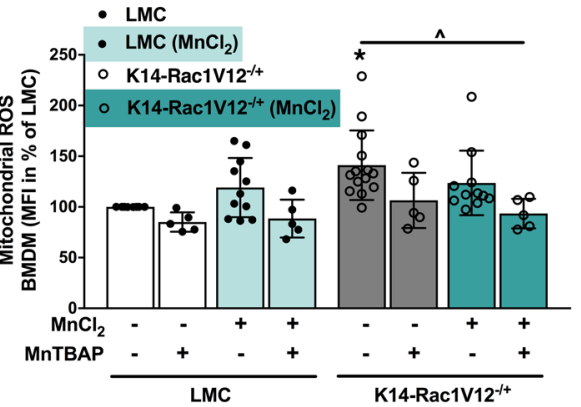

LMC

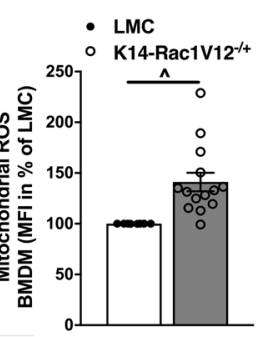

D
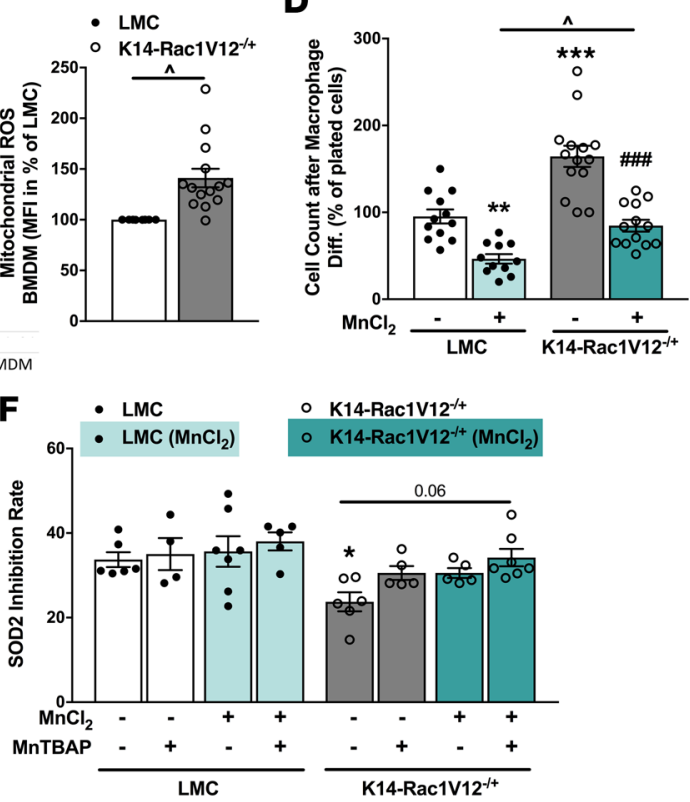

Figure 4. Manganese-dependent superoxide dismutase $\mathbf{2}$ (SOD2) function is impaired in psoriatic macrophages. (A) Mouse BM cell SOD2-derived ROS were labeled using MitoSox and examined by flow cytometry $(n=12 / 15)$. (B) qPCR analysis of BMDMs revealed changes in mitochondria-related gene expression $(n \geq 4)$. (C) Mitochondrial ROS of differentiated macrophages are increased in K14-Rac1V12-/+ BMDM. A representative histogram is shown $(n=12 / 15)$. (D) Differentiation levels of BMDMs in the presence of $\mathrm{MnCl}_{2}$, a SOD2 activator, was determined and significantly reduced ( $\left.n \geq 11\right)$. (E) mRNA expression profile was compared with pre- $\mathrm{MnCl}_{2}$ differentiation conditions using qPCR $(n \geq 5)$. (F) Since mitochondrial ROS staining does not qualify as an SOD2 activation measurement, SOD2 activity was detected using the SOD assay kit in the presence of potassium cyanide to inhibit SOD1 and SOD3 activity, displaying decreased activity in psoriatic BMDMs, which is rescued to LMC levels when differentiated with $\mathrm{MnCl}_{2}$ and treated with MnTBAP ( $n \geq$ 5). ( $\mathbf{G}$ and $\mathbf{H}$ ) Mitochondrial ROS were labeled using MitoSox after treatment with the SOD2 mimetic MnTBAP (12.5 $\mu \mathrm{M})$ in $\mathrm{MnCl}_{2}$ and control-differentiated LMC and psoriatic BMDM. Mitochondrial ROS were visualized using microscopy after PFA fixation and DAPI nuclei labeling $(n=3)$ and detected using flow cytometry $(n \geq 5)$. A representative histogram is shown. (Data are expressed as mean \pm SEM; ${ }^{*} P<0.05,{ }^{* *} P<0.005,{ }^{* * *} P<0.0005$ indicates significance to LMC control; ${ }^{\#} P<0.05$ indicates significance to $K 14-R a c 1 V 12^{-1+}$ control; ${ }^{\wedge} P<0.05$ indicates significance between indicated groups; [A, B, C, D, and F] Mann-Whitney test, [E and G] 2-way ANOVA with Bonferroni correction $P=0.01$, scale bar: $50 \mu \mathrm{m})$ (LMC, littermate control; MnTBAP, Manganese [III] Tetrakis [4-Benzoic Acid] Porphyrin chloride). 
A

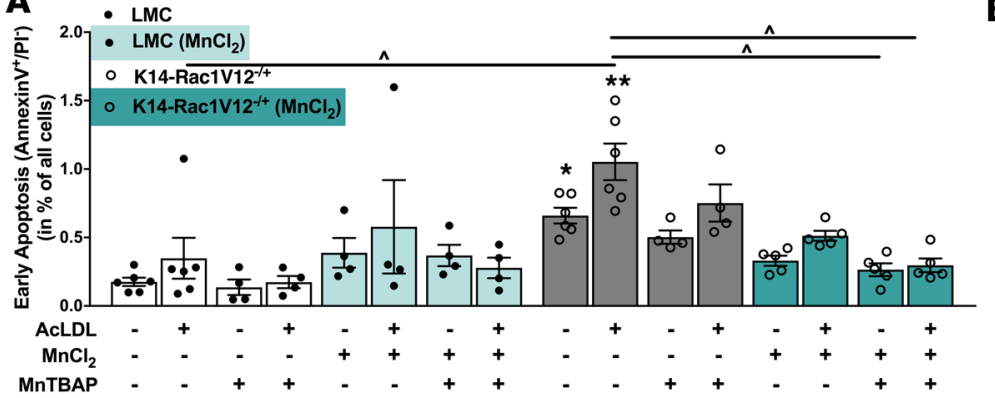

\section{B}

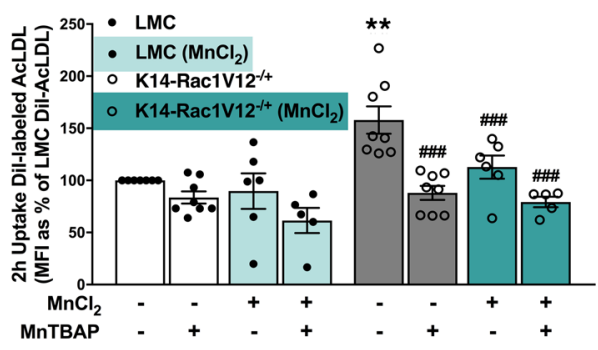

C

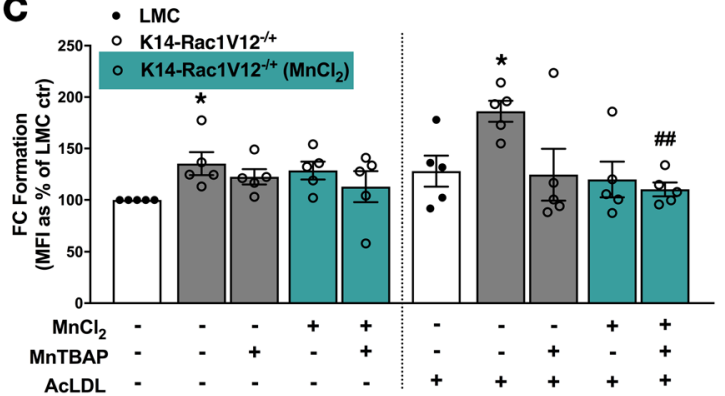

D

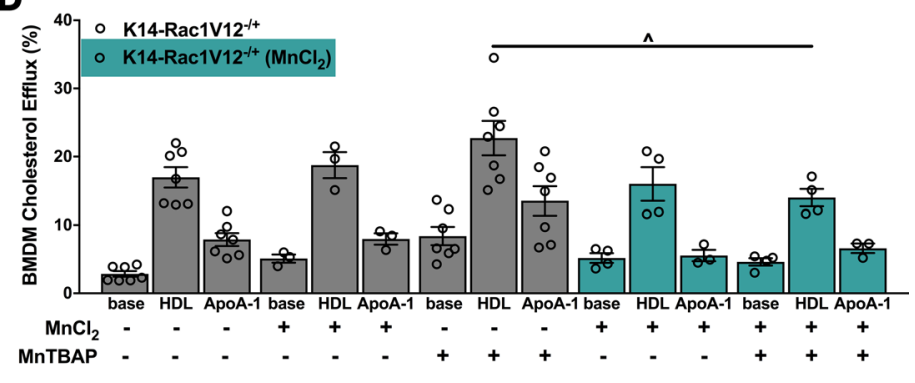

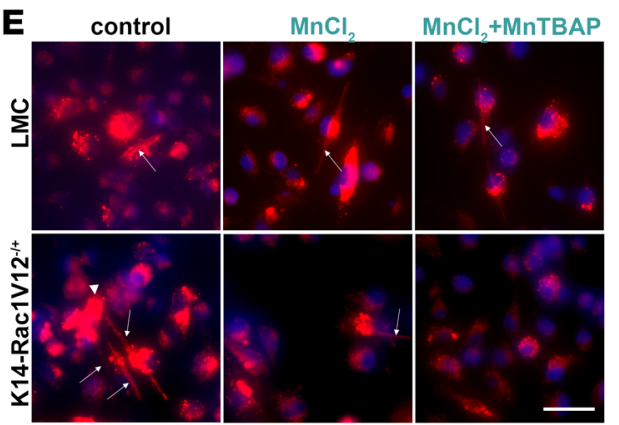

F

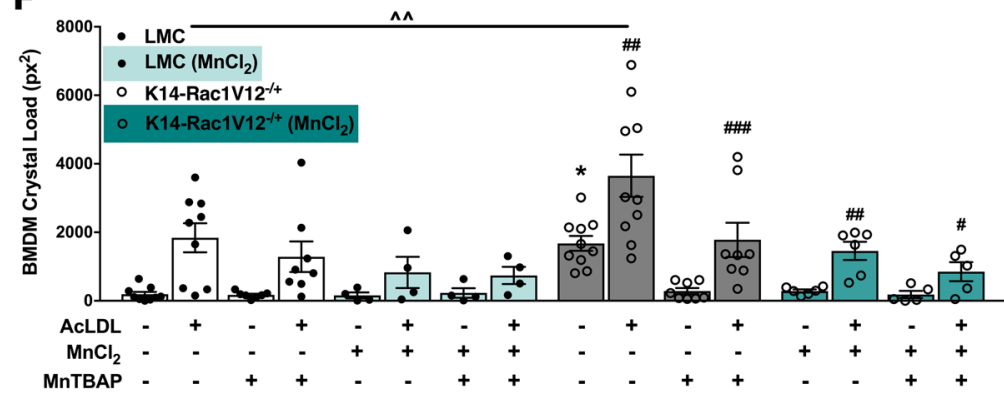

Figure 5. Manganese-dependent superoxide dismutase 2 (SOD2) is a key regulator in psoriasis-induced proatherosclerotic macrophage reprogramming. BMDM were isolated from $L M C$ and $K 14-R a c 1 V 12^{-/+}$mice. Cells were differentiated in the absence or presence of $5 \mu M \mathrm{MnCl}_{2}$. Additionally, sets of BMDMs were pretreated with $12.5 \mu \mathrm{M}$ MnTBAP, a SOD2 mimetic, before conducting the experiment. (A) Apoptosis and necrosis in BMDMs was determined using flow cytometry. Results of early apoptotic events under baseline and $50 \mu \mathrm{g} / \mathrm{ml} \mathrm{AcLDL-treated} \mathrm{conditions} \mathrm{(24h)} \mathrm{displays} \mathrm{a} \mathrm{significant}$ reduction in K14-Rac1V12 ${ }^{-/+}$BMDMs differentiated in the presence of Manganese ( $\left.n \geq 4\right)$. Modified lipid uptake (B), foam cell formation (C), and cholesterol efflux (D) experiments were performed in the presence of $\mathrm{MnCl}_{2}$ or $\mathrm{MnTBAP}_{\mathrm{N}}$ using control and $\mathrm{MnCl}_{2}$ differentiated BMDMs ( $n \geq 5$ ). (E) BMDMs were treated with $50 \mu \mathrm{g} / \mathrm{ml}$ Dil-AcLDL (red) for $24 \mathrm{~h}$ and nuclei labeled with DAPI (blue) to visualize AcLDL cholesterol crystal formation (arrows indicated CC, arrowheads indicate large densely lipid packed intracellular areas, representative images of total $n=3$; scale bar: $50 \mu \mathrm{m})$. (F) CC were visualized with a polarized light microscope and quantified using Image) under baseline and $50 \mu \mathrm{g} / \mathrm{ml}$ AcLDL treatment conditions ( $n \geq 5$ ). (Data are expressed as

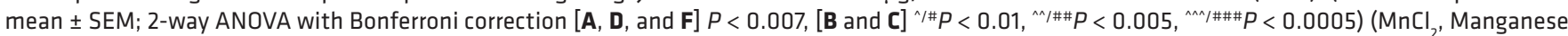
chloride; LMC, littermate control; MnTBAP, Manganese [III] Tetrakis [4-Benzoic Acid] Porphyrin chloride; ROS, reactive oxygen species; FC, foam cell formation; Ac, acetylated; LDL, low-density lipoprotein; MFI, median fluorescence intensity).

ports atherogenesis. An additional possibility explaining the decreased levels of circulating monocytes could be due to inflammation-induced changes in BM kinetics. We show that $\mathrm{CD} 45^{+}$cell numbers are increased in the BM of psoriasis mice, while circulating monocyte levels are decreased. Alterations in BM cell polarization into other cell types rather than monocytes/macrophages might be at play and should be investigated in future experiments.

Increased attraction and homing of inflammatory monocytes within the vessel wall is explained, in part, by the increased presence of chemoattractants like MCP-1, indicating an increased potential of transendothelial migration of psoriatic monocytes. Additionally, we speculate that alterations of CD62L (L-selectin) expression on circulatory blood cells - a marker shown to regulate accelerated adherence, transmigration, and homing of mononuclear cells (31-33) - might be altered, as well, in psoriasis. Future studies should consider the specific impact of the psoriatic serum on healthy and diseased endothelium. 
Vessel wall-infiltrating monocytes are precursors of atherosclerotic plaque macrophages. Despite the emerging thought that smooth muscle cell-to-monocyte conversion may occur (34), immune cell infiltration into the vessel wall is still highly likely. It is very likely that both mechanisms are of high importance for vascular inflammation and atherosclerosis development and progression. In both scenarios, macrophages and macrophage-like cells are of tremendous significance for atherosclerosis progression and regression $(30,35)$.

Commonly, macrophages are divided as antiinflammatory (M2) and proinflammatory (M1) macrophages. Macrophages are known to adapt to their surroundings and display plasticity, changing to the pathological needs of the disease and tissue $(36,37)$. Separation of macrophages according to their function might be the more proper classification method for different disease states (38). We demonstrate that psoriasis as a skin disease pushes macrophage function toward a proatherosclerotic phenotype, rather than the classical M1/M2 classification. Genes, described in the past as classical M1 genes in mice (e.g., Inos, Arg1) (36), have recently been characterized as alternative macrophage markers that do not fit the classic profile. Analysis of our mouse psoriatic macrophage gene expression for these recently described genes (39) displayed an alternative macrophage subset with few classical M1 genetic markers. Moreover, on the scanning electron microscope, psoriatic macrophages show a more flattened and round shape as it has been described for M1 macrophages (22). However, even though morphological similarities between psoriatic and M1 differentiated macrophages were present, their phenotype and gene expression showed major differences (data not shown), supporting our conclusion of an alternative psoriasis macrophage with pro-atherosclerotic characteristics. A proatherosclerotic macrophage is generally expected to exhibit increased lipid uptake, increased foam cell formation and decreased reverse macrophage cholesterol transport, which were seen in our PBMC-derived human macrophages and partially in the psoriatic mouse macrophage. These observed differences are most likely a result of three variants: a difference in species (human vs. mouse) (40); the origin of derived macrophages, which has been highlighted in the literature $(40,41)$ (BM versus peripheral blood); and differences in the differentiation process (adhesion versus cytokine/chemokine induced differentiation).

Chronic inflammation in psoriasis contributes to differences in macrophage differentiation and function. Cytokines elevated in psoriasis patients include TNF $\alpha, I L 12 / 23, I L 1 \beta, I F N \gamma$, and IL17 (42) and are often targeted in a psoriasis treatment regimen. These cytokines have been demonstrated to be of crucial importance for macrophage plasticity in acute or chronic aspects and in regard to atherogenesis $(11,19,43,44)$. However, since isolated macrophages in our study were differentiated under cytokine neutral conditions, a genetic preconditioning toward the proatherosclerotic phenotype may also modulate these characteristics. Recent understanding of epigenetic changes may be at play, as well. For example, in obstructive sleep apnea, the accumulation of CD36 macrophages within the aortic wall has been ascribed to histone modifications, ultimately accelerating CVD development (45). Clonal hematopoiesis and CVD have been brought to attention in a recent publication, where the authors report that the risk for CVD increases in patients carrying Tet2 mutations in their PBMCs (46). In terms of a possible involvement of changes in the micro RNA (miRNA) profile, the comparison of 2 recent reports makes miR223 a likely candidate to be involved in psoriasis-induced macrophage differentiation changes $(47,48)$. Finally, psoriasis itself has shown to be associated with epigenetic changes (49).

Manganese-dependent SOD2 is a key regulator of oxidative stress, counteracting toxic ROS. Impaired SOD2 function or expression has been reported to be critical in maintaining macrophage cholesterol homeostasis (50) and accelerating atherosclerosis development (51), and it is to be implicated in psoriasis development (17). Therefore, we speculated that SOD2 could be a key regulator in the observed macrophage dysfunction in psoriasis, resulting in accelerated atherogenesis. Autophagy — a process responsible for removing oxidatively damaged organelles like mitochondria and promoting efflux of lipids from lipid loaded cells - is impaired by the presence of ROS, involving NLRP3 inflammasome activation and cell damage (52). Moreover, impacted autophagy (53) has been shown to accelerate atherogenesis. Furthermore, CC treatment of macrophages has been shown to activate the NLRP3 inflammasome and accelerate atherosclerosis development (54), while endothelial-derived CC formation at very early stages of atherogenesis seems to be an initiating factor for atherosclerosis development (55). While we did not observe NLRP1 or NLRP3 inflammasome activation in psoriatic macrophages under our experimental conditions, a protective effect of SOD2 on efficiency of autophagy independently of NLRP1/3 inflammasome activation cannot be excluded.

The exact mechanism regarding how and where CC are formed is still under intense investigation. Enhanced lipid uptake and decreased efflux in psoriatic macrophages results in the enhanced accumulation of lipids. We speculate that impaired autophagy in psoriatic macrophages accelerates CC formation due to decreased SOD2 activity as a result of increased ROS presence. Lastly, it has recently 
been published that cholesterol crystallization can occur within lipid droplets in liver cells (56). The increased presence of lipid droplets within K14-Rac1V12-/+ BMDMs suggests a possible additional source of macrophage CC formation enhancing macrophage cell death and accelerating atherosclerosis development in psoriasis mice and patients.

Limitations of our study include the relatively small number of human cells studied. Furthermore, rare use of topical antibiotic therapy on very severe $\mathrm{K} 14-\mathrm{Rac} 1 \mathrm{~V} 12^{-/+}$mice may have impacted vascular disease progression. We also did not study patterns of thrombosis in this mouse model, which certainly should be done in the future $(7,8)$. Furthermore, our use of a less-utilized atherosclerosis mouse model $\left(\right.$ Srb1 $\left.1^{-/-} / A p o E R 61^{H / H}\right)$ instead of $A p o E^{-/-}$or $\mathrm{Ldlr}^{-/}$may limit generalizability of our findings. However, given the impact of Ldlr and ApoE on macrophage function and the psoriasis phenotype, our choice provided the best available option.

Future directions of our research include the treatment of the murine model with manganese (activation of SOD2) and MnTBAP, to replenish SOD2 itself in order to yield further insight into the role of SOD2 in psoriasis-driven atherosclerosis. Additionally, we seek to identify downstream pathway alterations of SOD2 to further understand cellular signaling and potentially develop refined future therapies. In conclusion, our results provide strong evidence that the presence of chronic, low-grade inflammation modulates key enzymes in macrophages, which may contribute to CC formation and defective lipid shuttling, both ultimately accelerating atherosclerosis development and progression.

\section{Methods}

Mice. K14-Rac1 mice sperm (strain CBA/CaJ) were provided by Peter Marinkovich (Stanford University) (9). The transgenic mice were rederived at Charles River with an CBA/CaCrl female for transfer to the NIH. Constitutively active V12Rac1 (Myc-tagged) was overexpressed in the basal keratinocytes of these mice using the keratinocyte-specific promoter 14 (K14). Furthermore, homozygous expression of K14Rac1V12 displays an extremely severe phenotype; therefore, we utilized only heterozygous mice for all experiments. Mice from the same litters lacking the expression of V12 were used as LMC. Rac1V12 mice were housed in standard cages, in a temperature-controlled, pathogen-free room with normal dark and light cycles on a normal chow diet. Mice were euthanized via $\mathrm{CO}_{2}$ asphyxiation, immediately followed by cervical dislocation at various weeks of age for organ harvesting. Due to the severity of this mouse model, ACUC required the topical application of antibacterial ointment locally on severely affected animals to avoid infection or sepsis and to ensure the well-being of the mice. Severity of psoriasis mice was determined following a 5-point list of visible symptoms summarized in Supplemental Table 1.

Substances and treatments. To examine the role of SOD2 within our experiments, cells were pretreated with $12.5 \mu \mathrm{M}$ MnTBAP (AbCam) for 1 hour and/or differentiated in the presence of $5 \mu \mathrm{M} \mathrm{MnCl}_{2}$ (MilliporeSigma).

Blood/plasma collection. Mouse blood samples were collected both via retroorbital bleeding and cardiac puncture, and they were transferred into EDTA Eppendorf tubes. Plasma was obtained by centrifugation at $1,500 \mathrm{~g}$ at $4^{\circ} \mathrm{C}$ for 15 minutes, and stored frozen at $-80^{\circ} \mathrm{C}$. Human blood samples were collected in CPT tubes (BD Biosciences), and PBMCs were isolated by centrifugation at $1,590 \mathrm{~g}$ for 20 minutes at room temperature.

Plasma lipid analyses and plasma cholesterol efflux measurements. Lipoproteins were fractionated by FPLC. Plasma lipoproteins from $100 \mu$ of pooled mouse plasma $(n=3-6)$ were separated by gel filtration utilizing 2 Superose 6 HR 10/30 columns (Pharmacia-LKB Biotechnology Inc.) connected in series. Lipids in the recovered fractions were assayed as described above. The elution volumes of the plasma lipoprotein separated by FPLC were VLDL, 15.0-16.0 ml; IDL/LDL, 20.0- 24.0 ml; and HDL, 30.0-31.0 ml. From unfractioned plasma total cholesterol (catalog 439-17501), free cholesterol (catalog 435-35801), phospholipids (catalog $433-$ 36201), and triglycerides (catalog 461-08992 and 461-09092) were detected enzymatically using commercially available kits (Wako Life Sciences) according to the manufacturer's recommendation. Plasma cholesterol efflux (HDL function) was measured by a validated ex vivo protocol as described previously (20).

Metabolic profiling of K14-Rac1V12-/+ mice. Open circuit calorimetry, activity, and food consumption data were collected using the Oxymax Comprehensive Laboratory Animal Monitoring System (Columbus Instruments). Mice were housed individually in the calorimetry cages for 5 days while metabolic parameters including maximum volume of oxygen $\left(\mathrm{VO}_{2}\right)$, maximum volume of carbon dioxde $\left(\mathrm{VCO}_{2}\right)$, and resting energy requirement (RER; $\mathrm{VCO}_{2} / \mathrm{VO}_{2}$ ) were measured by the system. Mice in the setup were fed using a spring-loaded feeder over top of a scale. A central hopper contained the food available for consumption by the animal; the hopper was surrounded by a concentric hopper, which scavenged any food spillage. This allowed for accurately measured grams of food consumed both cumulatively and per feeding episode. 
Plasma cytokine and chemokine detection. To measure plasma levels of proinflammatory cytokines and chemokines (e.g., mouse TNF $\alpha$, IL1 $\beta$, IL6, and CXCL1, chosen a priori) associated with CVD (43), plasma samples were collected from transgenic and LMC mice, and an enzyme-linked immunosorbent assay, V-PLEX Proinflammatory Panel 1 (mouse) Kit, was performed following manufacturer's protocol (Meso Scale Discovery). MCP-1 (CCL2) and chemokine ligand 5 (CCL5, RANTES) were detected in mouse plasma of adult LMC and K14-Rac1V12-/+ mice using the R\&D ELISA kits, MJE00 and MMR00, respectively.

Cell composition of human and mouse blood. Mouse blood was collected in EDTA-coated Eppendorf tubes. Whole blood (100 $\mu \mathrm{l})$ was transferred to a FACs tube and RBCs were lysed with $2 \mathrm{ml} \mathrm{ACK}$ lysis buffer for 15 minutes at room temperature. If RBCs remained, cells were lysed a second time. Cells were then washed twice with FACs buffer ( $1 \times$ PBS, 0.1\% BSA, $0.02 \%$ EDTA) and incubated for 10 minutes in $100 \mu \mathrm{l}$ of FcR Block (1:10, Miltenyi Biotec, catalog 130-092-575) at $4^{\circ} \mathrm{C}$. Without washing, $100 \mu 1$ of the 9-color antibody cocktail (Supplemental Table 2) was added to sample and incubated for 20 minutes at $4^{\circ} \mathrm{C}$. LIVE/DEAD Aqua fixable dye (Invitrogen, catalog L34957) was used to target viable cells. Cells were then washed and fixed in 1\% PFA and analyzed on the BD LSR II Flow Cytometer (BD Biosciences). Data were analyzed using FlowJo software (Tree Star Inc.) with a sequential gating scheme. Single cells were gated first using forward and side scatter; live cells were then identified and used for downstream gating scheme, as described in Supplemental Figure 7.

Cell composition of aorta, spleen, BM, and lymph node. K14-Rac1V12 ${ }^{-{ }^{+}}$and their LMC mouse aortas at 12-17 weeks of age were thoroughly cleaned from surrounding fat tissue and tunica adventitia and cut in $1-\mathrm{mm}$ rings. Digestion of aortic rings and lymph nodes to single-cell suspension was achieved using 1.5 Wünsch units Liberase (Roche Diagnostic) for 1 hour at $37^{\circ} \mathrm{C}$ under gentle rocking conditions. Spleens and $\mathrm{BM}$ of mice were separated into a cell suspension using a $50-\mu \mathrm{m}$ cell filter (catalog 22363547 , Thermo Fisher Scientific). All single cell suspensions were treated and analyzed as described above using the antibody cocktail in Supplemental Table 3.

$q P C R$. Total RNA was isolated from BMDMs and HMDM by TRIzol (Invitrogen). RNA was isolated using the Direct-zol RNA MiniPrep kit (Zymo Research Corp.) according to the manufacturer's protocol. Complementary DNA was created using a RT2 First Strand Kit (Qiagen), and qPCR analysis was performed using target-specific primer pairs (Eurofins Genomics, Supplemental Table 4) on the LightCycler 96 (Roche Diagnostics) using SYBR Green for visualization. GAPDH was used as a housekeeping gene to normalize mRNA levels.

$B M D M$ and HMDM culture. BMDMs were cultured from the BM of female and male K14-Rac1V12 ${ }^{-/+}$ and LMC mice aged 12-17 weeks as described previously (57). For experiments involving $\mathrm{MnCl}_{2}$, portions of the macrophages were differentiated for 6 days and replated in the presence of $5 \mu \mathrm{M} \mathrm{MnCl}_{2}$.

Several key experiments were performed using human monocyte-derived macrophages (HMDM) derived from PBMCs isolated from fresh donor blood via the attachment method. Briefly, PBMCs were isolated from CPT tubes via centrifugation at 1,590 $\mathrm{g}$ for 20 minutes at room temperature and washed with PBS 3 times. Cells were counted and plated at 500,000 cells $/ \mathrm{cm}^{2}$ in X-Vivo 15 media (Lonza) with $20 \%$ FBS. Cells were allowed to attach for 1 hour at $37^{\circ} \mathrm{C}$ and $5 \% \mathrm{CO}_{2}$ with a subsequent media change. Attached cells were allowed to differentiate for 5 days and were used for experiments without replating, except for HMDM efflux assays where replating at equal cell numbers is important for accurate experimental procedure. For experiments involving $\mathrm{MnCl}_{2}$, portions of the macrophages were differentiated for 5 days in the presence of $5 \mu \mathrm{M} \mathrm{MnCl}_{2}$.

Lipid uptake, crystal formation, and foam cell formation assay. For modified LDL lipid uptake assays, human and mouse macrophages were treated with $20 \mu \mathrm{g} / \mathrm{ml}$ DiIAcLDL and DiIOxLDL (AlfaAeser) for 2 or 4 hours, fixed using 4\% PFA in PBS for 10 minutes at room temperature, nuclei labeled with DAPI, and mounted in DAKO fluorescence mounting media.

For crystal formation and foam cell formation assays, macrophages were treated for 24 hours with 50 $\mu \mathrm{g} / \mathrm{ml}$ AcLDL or OxLDL. Afterward, cells were fixed using 4\% PFA in PBS for 10 minutes at room temperature, subjected to polarized light microscopy, and then stained using Bodipy493/503 (Thermo Fisher Scientific) for 30 minutes at room temperature.

For uptake and foam cell formation fluorescence, 5 pictures per treatment condition were taken at equal exposure times, and MFI per cell was detected using ImageJ. Data were analyzed using Microsoft excel and PRISM. The results of BMDM experiments were confirmed using flow cytometry detecting MFIs using the LSR Fortessa (BD Biosciences). 
Detection of free and esterified cholesterol in macrophages. Mouse BMDMs were incubated with $50 \mu \mathrm{g} / \mathrm{ml}$ AcLDL for 24 hours to allow cholesterol loading and foam cell formation. For flow cytometry analysis, cells were deattached afterward, using Cell Stripper (Corning), and they were fixed with 4\% PFA at room temperature for 10 minutes. As an indicator for free cholesterol, cells were labeled for 2 hours at room temperature using $0.05 \mathrm{mg} / \mathrm{ml}$ Filipin in PBS containing 10\% FBS after a 10-minute incubation with 1.5 $\mathrm{mg} / \mathrm{ml}$ glycine/PBS. Bodipy493/505 $(10 \mu \mathrm{g} / \mathrm{ml})$ in PBS for 30-minute labeling was used as an indicator for lipid droplet loading of macrophages. MFI per cell was detected using the LSR Fortessa (BD Biosciences).

Polarized light microscopy. HMDM and BMDMs were fixed using 4\% PFA in PBS. Fixed cells and $10-\mu \mathrm{m}$ frozen sections of the aortic root were imaged using a polarized light microscope (Olympus IX81F-2 with IPLab imaging software).

Macrophage efflux assay. Human and mouse macrophage capacity to efflux uptaken radioactive cholesterol toward standardized HDL and ApoA-1 was performed as described previously $(57,58)$.

Western blot analysis. To determine skin-specific expression of Rac1, Myc, and other markers (Supplemental Table 5), Western blot analysis of various organs and BMDMs was performed as described previously (59). Briefly, organs and cells were lysed with Cell Lytic M (MilliporeSigma, plus proteinase and phosphatase inhibitors) on ice. Protein concentrations of lysates were determined using the Pierce BCA Protein Kit (Thermo Fisher Scientific). Equal protein amounts were used for polyacrylamide gel electrophoresis, with subsequent Western blot transfer. After blocking and antibody incubation (Supplemental Table 5), specifically sized protein bands were visualized and analyzed using Licor Imaging Studio software (Licor, Bioscience).

Apoptosis assay. BMDM were subjected to AcLDL and OxLDL treatment for 24 hours, harvested using Cell Stripper, and stained for AnnexinV/PI using an apoptosis/necrosis staining kit (BD Biosciences) according to the manufacturer's recommendations. Cells were analyzed using LSR Fortessa (BD Biosciences) using FACSDiva and FlowJo software.

Scanning electron microscopy. Samples for scanning electron microscopy were prepared as described previously $(55,59)$ and imaged using a Hitachi S-3400N1 scanning electron microscope at the Electron Microscopy Core Facility, NHLBI. Per sample, 5 independent pictures were taken at comparable magnifications. Afterward, the number of round and elongated macrophages per image were counted and analyzed using PRISM software.

TEM. Mouse aortas were harvested and thoroughly cleaned. The part adjacent to the left subclavian artery was immediately fixed as described previously $(55,59)$. BMDMs were isolated and treated as described above. Cells were fixed and prepared as described previously (59). Ultra-thin section samples were imaged using a JEM1400 transmission electron microscope at the Electron Microscopy Core Facility, NHLBI.

Fluorescence microscopy staining of cells and tissue. Cells were washed and fixed using 4\% PFA in PBS for 10 minutes at room temperature ( $\mathrm{pH} 7.4$ ), permeabilized using $0.1 \%$ TritonX-100 in PBS for 5 minutes at room temperature, and blocked in 2\% BSA/10\% NGS for 30 minutes at room temperature. Primary antibodies (Supplemental Table 5) were added at a 1:100 dilution in 2\% BSA/PBS and incubated overnight at $4^{\circ} \mathrm{C}$. The following day, cells were thoroughly washed and fluorescently labeled by a secondary antibody (in $2 \%$ BSA/PBS) for 1 hour at room temperature in the dark. DAPI was added to label the nuclei, and cells were mounted using DAKO fluorescent mounting media. Skin samples and mouse hearts were fixed in $4 \% \mathrm{PFA} / 5 \%$ sucrose in $\mathrm{PBS}$ overnight at $4^{\circ} \mathrm{C}$ and, afterward, transferred into $30 \%$ sucrose for 3 hours for further dehydration. After embedding in Tissue Tek and storing at $-80^{\circ} \mathrm{C}$, tissue samples were cryo sectioned into $10-\mu \mathrm{m}$ sections. H\&E staining was routinely performed following the manufacturer's recommendations (MilliporeSigma). For immunolabeling, tissues were treated using antigen citrate buffer/heat or trypsin antigen retrieval (Supplemental Table 5). Afterward, sections were blocked and stained as described above. Images were taken using a Zeiss Axiovert Microscope with equal exposure times to allow for further analysis using ImageJ software. Five images per sample per experiment were analyzed.

MitoSOX activity assay. Human and mouse macrophages and their precursors mouse BM cells and human PBMCs were treated as indicated in Figure 4, A, C, G, and H, and Supplemental Figure 6, A and D. Afterward, cellular mitochondrial ROS were labeled using 2.5 $\mu \mathrm{M}$ MitoSOX (Thermo Fisher Scientific) for 15 minutes at $37^{\circ} \mathrm{C} 5 \% \mathrm{CO}_{2}$ in HBSS without serum or FBS. Labeled cells were harvested, fixed using $1 \%$ PFA in flow buffer, and analyzed using the BD Fortessa. Cells were initially gated and separated from debris. MFI of single cells was determined and analyzed using Excel and PRISM software. 
In a separate experiment, BMDMs were replated on 8-well glass chamber slides (MilliporeSigma), treated, and labeled using MitoSOX. Cells were fixed using 4\% PFA, and nuclei were labeled using DAPI and imaged using an Axiovert microscope at equal exposure times.

Detection of SOD2 activity. BMDM were differentiated with and without addition of $\mathrm{MnCl}_{2}$, replated at equal cell numbers $\left(1 \times 10^{6}\right.$ cells/12 well), and treated with control or MnTBAP containing media for 24 hours in the presence or absence of $\mathrm{MnCl}_{2}$. Afterward, cells were lysed (0.1 M Tris/ $\mathrm{HCl}, \mathrm{pH}$ 7.4, $0.05 \%$ TritonX-100, $5 \mathrm{mM} \beta-\mathrm{ME}, 0.1 \mathrm{mg} / \mathrm{ml} \mathrm{PMSF}$ ) and centrifuged for 5 minutes at $4^{\circ} \mathrm{C}$ at $14,000 \mathrm{~g}$. Supernatants were subjected to the SOD activity kit (AbCam) according to manufacturer's recommendations in the presence of $5 \mu \mathrm{M}$ potassium cyanide to inhibit SOD1 and SOD3 activity. As an internal control, a SOD1 (AbCam) positive control was assayed in the presence and absence of potassium cyanide.

Mouse atherosclerosis study. To create an atherosclerosis susceptible psoriatic mouse phenotype, K14-

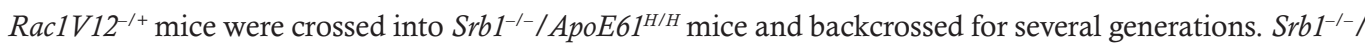
ApoE6 $1^{H / H}$ mice $(60,61)$ were a gift from Allen T. Remaley (NHLBI, NIH). Female Srb1 $1^{-/-} / A p o E 61^{H / H} / K 14$ $\mathrm{Racl}^{-/+}$and $\mathrm{Srb1} 1^{-/-} / \mathrm{ApoER} 61^{\mathrm{H} / \mathrm{H}} / \mathrm{K} 14-\mathrm{Rac1} \mathrm{V12^{-/+ }}$ mice 8-10 weeks of age were fed a HFD containing $15.8 \%$ (wt/wt) fat and 1.25\% cholesterol (diet 94059; Harlan Teklad) for 2 weeks. Female mice only were used for the atherosclerosis study due to limitations presented in the Srb1-breeding scheme. Psoriasis severity was determined before the start of HFD and at sacrifice. At sacrifice, lymph node and spleen samples were collected for mRNA and flow analysis. Blood was sampled in EDTA tubes for flow analysis of cell composition, and plasma was separated for lipid and cytokine analysis. Aortas and hearts were used for quantification and immunohistological analysis of atherosclerosis. The extent of atherosclerosis was detected in aortas and aortic root sections by staining with Oil Red $\mathrm{O}$ and H\&E. Plaque areas were measured using ImageJ software, as described previously (59). Subsequently aortic root sections were used for analysis of macrophage, smooth muscle cell and CC (polarized light [PL] microscopy) content, necrotic core area, and collagen content by Picro Sirius Red staining with subsequent PL microscopy (59). Movat and van Kossa staining were performed to visualize elastin/collagen and calcification, respectively.

Plasma lipid analyses after HFD. Total cholesterol (catalog 10007640) and total triglyceride (catalog 10010303) levels of 6-hour fasting LMC and K14-Rac1V12-/+ mice were measured using kits from Cayman Chemicals following the manufacturer's recommendation.

Statistics. Statistical comparison between groups was performed using PRISM 7.0 (GraphPad) software. Data are represented as mean \pm SEM. Statistical significance was evaluated using according statistical tests. Comparison of 2 groups was performed using Mann-Whitney test. Significance was established at a $\mathrm{p}$ value of $P<0.05$. ( ${ }^{*} P<0.05,{ }^{* *} P<0.005,{ }^{* * *} P<0.0005$ indicates significance to LMC control; ${ }^{\wedge} P<0.05,{ }^{\wedge} P<$ $0.005,{ }^{\wedge} P<0.0005$ significance between indicated groups, ${ }^{\sharp} P<0.05$, ${ }^{\# \#} P<0.005$, ${ }^{\# \#} P<0.0005$ indicates significance to $\mathrm{K} 14-\mathrm{Rac} 1 \mathrm{~V} 12^{-/+}$untreated control). In cases of multiple parameter comparison, 2-way ANOVA statistical test with subsequent Bonferroni correction was performed. All experiments were performed with at least an independent experiment number of $n=3$ and, where possible, in duplicates or triplicates.

Study Approval. The present study involving human and murine studies underwent all necessary institutional approvals and review boards.

Human subjects: Study approval was obtained from the IRB) at NHLBI, NIH in accordance with the principles of Declaration of Helsinki. All guidelines for good clinical practice and those set forth by the NIH Radiation Safety Commission and in the Belmont Report (National Commission for the Protection of Human Subjects of Biomedical and Behavioral Research) were followed. All study participants in the cohort provided written informed consent. All the participants were adequately compensated. Data for all psoriasis patients under the cohort study at NHLBI/NIH were obtained under a protocol titled Psoriasis, Atherosclerosis and Cardiometabolic Disease Initiative (PACI) (13-H-0065). Data from healthy volunteers were obtained under a separate study protocol subjected to the same rules as the PACI study (13-H-0194).

Murine studies: Murine study approval was obtained from the NIH/NHLBI ACUC with the according protocol number H-0275R1. Mouse handling and housing was conducted according to proper IACUC and NIH ASP regulations and guidelines.

\section{Author contributions}

YB and NNM designed the study. YB, QN, AAJ, and NNM wrote the manuscript. YB planned, performed and analyzed most experiments. QN and GS performed experiments and maintained the mouse colony. HLT and JIS performed blood flow and analysis. PKD analyzed organ flow experiments. CHL, AVS, NLV, SMR 
and MPP performed experiments. MCGW and MPM provided reagents, advice and revised the manuscript. $\mathrm{AKD}, \mathrm{ADB}$ and $\mathrm{AG}$ performed human and mouse regression analysis. JAR coordinated the human sample collection. DAS, CKEB, TCL and ZXY helped with experiments as part of NHLBI Core facilities. JMG and HSK critically reviewed the manuscript. MPP, JMG, HSK and NNM were excellent mentors and supervisor.

\section{Acknowledgments}

The authors are grateful for the contributions of our clinical and imaging team of the NIH Clinical Center, the NHLBI Electron Microscopy Core and the NHLBI Flow Cytometry Core. Special thanks is given to Dr. Yu and staff from the NHLBI Pathology Core as well as the NIH NIAD Animal Facility under the direction of Dr. James 'Buster' Hawkins. The first author Y. Baumer is supported by the NPF-NIH Robertson Fellowship awarded by the National Psoriasis Foundation. The senior author N.N. Mehta is supported by intramural grant HL006193-04. M.P. Marinkovich's support on this work was from the U.S. Department of Veterans Affairs Office of Research and Development; the National Institute of Arthritis and Musculoskeletal and Skin Diseases (NIH grant AR47223) and the National Psoriasis Foundation. J.M. Gelfand was supported by NIAMS (K24 AR 064310).

Address correspondence to: Nehal N. Mehta, Section of Inflammation and Cardiometabolic Diseases, National Heart, Lung and Blood Institute, 10 Center Drive, Clinical Research Center, Room 5-5140, Bethesda, Maryland 20892, USA. Phone: 301.827.0483; Email: nehal.mehta@nih.gov.

1. Rachakonda TD, Schupp CW, Armstrong AW. Psoriasis prevalence among adults in the United States. J Am Acad Dermatol. 2014;70(3):512-516.

2. Gelfand JM, Neimann AL, Shin DB, Wang X, Margolis DJ, Troxel AB. Risk of myocardial infarction in patients with psoriasis. JAMA. 2006;296(14):1735-1741.

3. Harrington CL, Dey AK, Yunus R, Joshi AA, Mehta NN. Psoriasis as a human model of disease to study inflammatory atherogenesis. Am J Physiol Heart Circ Physiol. 2017;312(5):H867-H873.

4. Gudjonsson JE, Johnston A, Dyson M, Valdimarsson H, Elder JT. Mouse models of psoriasis. J Invest Dermatol. 2007;127(6):1292-1308.

5. Wagner EF, Schonthaler HB, Guinea-Viniegra J, Tschachler E. Psoriasis: what we have learned from mouse models. Nat Rev Rheumatol. 2010;6(12):704-714.

6. Johnston A, et al. Keratinocyte overexpression of IL-17C promotes psoriasiform skin inflammation. J Immunol. 2013;190(5):2252-2262.

7. Wang Y, et al. Chronic skin-specific inflammation promotes vascular inflammation and thrombosis. J Invest Dermatol. 2012;132(8):2067-2075.

8. Golden JB, et al. Chronic, not acute, skin-specific inflammation promotes thrombosis in psoriasis murine models. J Transl Med. $2015 ; 13: 382$.

9. Winge MC, et al. RAC1 activation drives pathologic interactions between the epidermis and immune cells. JClin Invest. 2016;126(7):2661-2677.

10. Geeraerts X, Bolli E, Fendt SM, Van Ginderachter JA. Macrophage Metabolism As Therapeutic Target for Cancer, Atherosclerosis, and Obesity. Front Immunol. 2017;8:289.

11. Cochain C, Zernecke A. Macrophages in vascular inflammation and atherosclerosis. Pflugers Arch. 2017;469(3-4):485-499.

12. Clark RA, Kupper TS. Misbehaving macrophages in the pathogenesis of psoriasis. J Clin Invest. 2006;116(8):2084-2087.

13. Mehta NN, Azfar RS, Shin DB, Neimann AL, Troxel AB, Gelfand JM. Patients with severe psoriasis are at increased risk of cardiovascular mortality: cohort study using the General Practice Research Database. Eur Heart J. 2010;31(8):1000-1006.

14. Voloshyna I, Mounessa J, Carsons SE, Reiss AB. Effect of inhibition of interleukin-12/23 by ustekinumab on the expression of leptin and leptin receptor in human THP-1 macrophages. Clin Exp Dermatol. 2016;41(3):308-311.

15. Nerlich A, et al. C/EBP $\beta$ is a transcriptional key regulator of IL-36 $\alpha$ in murine macrophages. Biochim Biophys Acta. 2015;1849(8):966-978.

16. Moore J. TREATMENT OF PSORIASIS BY MANGANESE. Br Med J. 1922;2(3210):41.

17. Löntz W, Sirsjö A, Liu W, Lindberg M, Rollman O, Törmä H. Increased mRNA expression of manganese superoxide dismutase in psoriasis skin lesions and in cultured human keratinocytes exposed to IL-1 beta and TNF-alpha. Free Radic Biol Med. 1995;18(2):349-355.

18. Fukai T, Ushio-Fukai M. Superoxide dismutases: role in redox signaling, vascular function, and diseases. Antioxid Redox Signal. 2011;15(6):1583-1606.

19. Zernecke A, Weber C. Chemokines in atherosclerosis: proceedings resumed. Arterioscler Thromb Vasc Biol. 2014;34(4):742-750.

20. Mehta NN, et al. Abnormal lipoprotein particles and cholesterol efflux capacity in patients with psoriasis. Atherosclerosis. 2012;224(1):218-221.

21. Salahuddin T, et al. Cholesterol efflux capacity in humans with psoriasis is inversely related to non-calcified burden of coronary atherosclerosis. Eur Heart J. 2015;36(39):2662-2665.

22. Bauer A, Subramanian N, Villinger C, Frascaroli G, Mertens T, Walther P. Megapinocytosis: a novel endocytic pathway. Histochem Cell Biol. 2016;145(6):617-627.

23. Rakkola R, Matikainen S, Nyman TA. Proteome analysis of human macrophages reveals the upregulation of manganese-con- 
taining superoxide dismutase after toll-like receptor activation. Proteomics. 2007;7(3):378-384

24. Lombard DB, Zwaans BM. SIRT3: as simple as it seems? Gerontology. 2014;60(1):56-64.

25. Naik HB, et al. Severity of Psoriasis Associates With Aortic Vascular Inflammation Detected by FDG PET/CT and Neutrophil Activation in a Prospective Observational Study. Arterioscler Thromb Vasc Biol. 2015;35(12):2667-2676.

26. Tawakol A, et al. In vivo $18 \mathrm{~F}$-fluorodeoxyglucose positron emission tomography imaging provides a noninvasive measure of carotid plaque inflammation in patients. J Am Coll Cardiol. 2006;48(9):1818-1824.

27. Tom WL, et al. Characterization of Lipoprotein Composition and Function in Pediatric Psoriasis Reveals a More Atherogenic Profile. J Invest Dermatol. 2016;136(1):67-73.

28. Rohatgi A, et al. HDL cholesterol efflux capacity and incident cardiovascular events. N Engl J Med. 2014;371(25):2383-2393.

29. Yona S, et al. Fate mapping reveals origins and dynamics of monocytes and tissue macrophages under homeostasis. Immunity 2013;38(1):79-91

30. Rahman K, et al. Inflammatory Ly6Chi monocytes and their conversion to M2 macrophages drive atherosclerosis regression. $J$ Clin Invest. 2017;127(8):2904-2915.

31. Rzeniewicz K, et al. L-selectin shedding is activated specifically within transmigrating pseudopods of monocytes to regulate cell polarity in vitro. Proc Natl Acad Sci USA. 2015;112(12):E1461-E1470.

32. Chen YS, et al. Involvement of L-selectin expression in Burkholderia pseudomallei-infected monocytes invading the brain during murine melioidosis. Virulence. 2017;8(6):751-766.

33. Biancone L, Cantaluppi V, Duò D, Deregibus MC, Torre C, Camussi G. Role of L-selectin in the vascular homing of periphera blood-derived endothelial progenitor cells. J Immunol. 2004;173(8):5268-5274.

34. Shankman LS, et al. KLF4-dependent phenotypic modulation of smooth muscle cells has a key role in atherosclerotic plaque pathogenesis. Nat Med. 2015;21(6):628-637.

35. Moore KJ, Sheedy FJ, Fisher EA. Macrophages in atherosclerosis: a dynamic balance. Nat Rev Immunol. 2013;13(10):709-721.

36. Sica A, Mantovani A. Macrophage plasticity and polarization: in vivo veritas. J Clin Invest. 2012;122(3):787-795.

37. Wynn TA, Chawla A, Pollard JW. Macrophage biology in development, homeostasis and disease. Nature. 2013;496(7446):445-455.

38. Nahrendorf M, Swirski FK. Abandoning M1/M2 for a Network Model of Macrophage Function. Circ Res. 2016;119(3):414-417.

39. Beyer M, et al. High-resolution transcriptome of human macrophages. PLoS ONE. 2012;7(9):e45466.

40. Mestas J, Hughes CC. Of mice and not men: differences between mouse and human immunology. J Immunol. 2004;172(5):2731-2738

41. Waldo SW, et al. Heterogeneity of human macrophages in culture and in atherosclerotic plaques. Am J Pathol. 2008;172(4):1112-1126

42. Arican O, Aral M, Sasmaz S, Ciragil P. Serum levels of TNF-alpha, IFN-gamma, IL-6, IL-8, IL-12, IL-17, and IL-18 in patients with active psoriasis and correlation with disease severity. Mediators Inflamm. 2005;2005(5):273-279.

43. Smith E, et al. Blockade of interleukin-17A results in reduced atherosclerosis in apolipoprotein E-deficient mice. Circulation 2010;121(15):1746-1755.

44. Butcher MJ, Gjurich BN, Phillips T, Galkina EV. The IL-17A/IL-17RA axis plays a proatherogenic role via the regulation of aortic myeloid cell recruitment. Circ Res. 2012;110(5):675-687.

45. Cortese R, et al. Aorta macrophage inflammatory and epigenetic changes in a murine model of obstructive sleep apnea: Potential role of CD36. Sci Rep. 2017;7:43648.

46. Jaiswal S, et al. Clonal Hematopoiesis and Risk of Atherosclerotic Cardiovascular Disease. N Engl J Med. 2017;377(2):111-121.

47. Liu Y, Liu Q. MicroRNAs as regulatory elements in psoriasis. Open Med (Wars). 2016;11(1):336-340.

48. Self-Fordham JB, Naqvi AR, Uttamani JR, Kulkarni V, Nares S. MicroRNA: Dynamic Regulators of Macrophage Polarization and Plasticity. Front Immunol. 2017;8:1062.

49. Gudjonsson JE, Krueger G. A role for epigenetics in psoriasis: methylated Cytosine-Guanine sites differentiate lesional from nonlesional skin and from normal skin. J Invest Dermatol. 2012;132(3 Pt 1):506-508.

50. Graham A. Mitochondrial regulation of macrophage cholesterol homeostasis. Free Radic Biol Med. 2015;89:982-992.

51. Ballinger SW, et al. Mitochondrial integrity and function in atherogenesis. Circulation. 2002;106(5):544-549.

52. Arora M, Kaul D. Coronary atherosclerosis: Significance of autophagic armour. World J Cardiol. 2012;4(9):271-274.

53. Razani B, et al. Autophagy links inflammasomes to atherosclerotic progression. Cell Metab. 2012;15(4):534-544.

54. Duewell P, et al. NLRP3 inflammasomes are required for atherogenesis and activated by cholesterol crystals. Nature. 2010;464(7293):1357-1361.

55. Baumer Y, et al. Hyperlipidemia-induced cholesterol crystal production by endothelial cells promotes atherogenesis. Nat Commun. 2017;8(1):1129.

56. Ioannou GN, et al. Cholesterol crystallization within hepatocyte lipid droplets and its role in murine NASH. J Lipid Res. 2017;58(6):1067-1079.

57. McCurdy S, Baumer Y, Toulmin E, Lee BH, Boisvert WA. Macrophage-Specific Expression of IL-37 in Hyperlipidemic Mice Attenuates Atherosclerosis. J Immunol. 2017;199(10):3604-3613.

58. Meiler S, Baumer Y, McCurdy S, Lee BH, Kitamoto S, Boisvert WA. Cluster of differentiation 43 deficiency in leukocytes leads to reduced atherosclerosis--brief report. Arterioscler Thromb Vasc Biol. 2015;35(2):309-311.

59. Baumer Y, et al. CD98 regulates vascular smooth muscle cell proliferation in atherosclerosis. Atherosclerosis. 2017;256:105-114

60. Zhang S, et al. Diet-induced occlusive coronary atherosclerosis, myocardial infarction, cardiac dysfunction, and premature death in scavenger receptor class B type I-deficient, hypomorphic apolipoprotein ER61 mice. Circulation. 2005;111(25):3457-3464.

61. Nakagawa-Toyama Y, Zhang S, Krieger M. Dietary manipulation and social isolation alter disease progression in a murine model of coronary heart disease. PLoS One. 2012;7(10):e47965. 